\title{
MALAT1 Induces Fibroblast Activation to Promote Cancer Progression via Blunting Autophagic Flux and Instigating IL-6 Secretion in Gastric Cancer Cells
}

\section{Zhen-qiang Wang}

Shanghai Jiao Tong University Medical School Affiliated Ruijin Hospital

\section{Xin-jing Wang}

Shanghai Jiao Tong University Medical School Affiliated Ruijin Hospital

Tian-qi Zhang

Shanghai Jiao Tong University Medical School Affiliated Ruijin Hospital

\section{Li-ping Su}

Shanghai Jiao Tong University Medical School Affiliated Ruijin Hospital

Bingya Liu

Shanghai Jiao Tong University Medical School Affiliated Ruijin Hospital

\section{Zheng-gang Zhu}

Shanghai Jiao Tong University Medical School Affiliated Ruijin Hospital

Chen Li ( $\sim$ leedoctor@sina.com )

Shanghai Jiao Tong University Medical School Affiliated Ruijin Hospital

\section{Research}

Keywords: gastric cancer, IncRNA MATLAT1, autophagy, fibroblast

Posted Date: October 9th, 2020

DOI: https://doi.org/10.21203/rs.3.rs-88665/v1

License: (c) (1) This work is licensed under a Creative Commons Attribution 4.0 International License. Read Full License 


\section{Abstract}

\section{Background}

Autophagy defection contributes to inflammation dysregulation, which plays an important role in gastric cancer (GC) progression. Various studies have demonstrated that long noncoding RNA could function as novel regulators of autophagy. However, the epigenetic regulatory mechanisms by which blockage of autophagy caused by long noncoding RNAs develops cancer progression remain unclear. This study aimed to investigate the role of the long noncoding RNA MALAT1 in the autophagy related inflammation dysregulation of GC progression and elucidate the underlying molecular mechanisms.

\section{Methods}

The effect of MALAT1 on autophagy in GC cells was analysed by immunoblotting, immunofluorescence and transmission electron microscopy. The role of MALAT1 in regulating

inflammation dysregulation was evaluated by ELISA, qPCR and immunoblotting. Bioinformatic prediction, RNA immunoprecipitation-qRCP and immunofluorescence were performed to identify the competitive binding relationship among MALAT1, ELAVL1 and PTEN 3'-UTRs.

The chromatin immunoprecipitation assay was performed to examine STAT3 interaction in the MALAT1 promoter region. Immunoblotting was performed to identify the PTEN/AKT/mTOR and SQSTM1/NF-kb mediated pathways altered by MALAT1. The influence of MALAT1 on fibroblasts activation were determined both in in vitro and in vivo.

\section{Results}

We found that the long noncoding RNA MALAT1 could promote interleukin-6 (IL-6) secretion in GC cells by blocking autophagic flux. Moreover, IL- 6 induced by MALAT1 could activate normal to cancerassociated fibroblast conversion. The interaction between GC cells and cancer-associated fibroblasts in the tumour microenvironment could facilitate cancer progression. Mechanistically, MALAT1 overexpression destabilized the PTEN mRNA in GC cells by competitively interacting with the RNA-binding protein ELAVL1 to activate the AKT/mTOR pathway for impairing autophagic flux. As the consequence of autophagy inhibition, SQSTM1 accumulation promotes NF-KB translocation to elevate IL-6 expression. Furthermore, STAT3 induced by IL-6 is responsible for upregulation of MALAT1 in GC.

\section{Conclusions}

Overall, these results demonstrated that intercellular interaction between GC cells and fibroblasts was mediated by autophagy inhibition caused by increased MALAT1 that promote GC progression, providing novel prevention and therapeutic strategies for GC.

\section{Background}


Inflammatory mediators within tumour microenvironment (TME) play important roles in promoting gastric cancer (GC) progression. The various cytokines within the GC TME are secreted from inflammatory cells, fibroblasts, and GC cells[1]. Moreover, GC cells could receive extracellular signals, which could further modulate TME via paracrine secretion of cytokine. The cross talk between GC cells and stroma cells facilitate cancer progression. Cancer-associated fibroblasts (CAFs), a major component of the tumour stroma, are a critical source of various molecules secreted in TME, which stimulate cancer cells progression. Similarly, the fluctuation of inflammatory mediators (growth factors, interleukin) by cancer cells in TME also altered resident fibroblast phenotypes and lead to normal fibroblast (NF) activation, considered as the main CAF source[2, 3]. Increasing evidence demonstrated interleukin-6 (IL-6) was abundant in GC TME, facilitating GC progression[4]. Most studies have reported that IL-6 could be released from CAFs and promote GC cells proliferation or metastasis in a paracrine way. However, IL-6 secretion from GC and effect on modulating TME has not been studied in detail. Here, we have found that autophagy inhibition in GC could upregulate IL-6 expression and secretion.

Autophagy is an important biological process that appears to be a double-edged sword with respect to cytokine signalling and modulating tumour progression in certain instances[5]. Activated autophagy could protect cells from inflammatory damage by inhibiting autophagy and aggravating inflammatory responses in many tissues $[5,6]$. It is widely accepted that autophagy defects contribute to inflammation and autophagy inhibition under the condition of chronic inflammation devoted to oncogenesis[7, 8]. Several reports have suggested that long noncoding RNA (IncRNA) could function as novel autophagy regulators. Silencing IncRNA-FA2H-2 facilitates impairment of oxidized low-density lipoprotein-induced autophagy flux to activate inflammation for increased IL-6 and other cytokine production[9]. Defective autophagy increases inflammatory mediator (such as TNF-a and HGF) production to promote hepatocellular carcinoma[7]. Impaired autophagy could promote chemoresistance in gastric cancer via IncRNA ARHGAP5-AS1 accumulation[10]. Although recent studies have shed light on some autophagy impairment mechanisms in $\mathrm{GC}$, the molecular components that mediate the process are yet to be fully identified.

LncRNA metastasis-associated lung adenocarcinoma transcript 1 (MALAT1) has been reported to activate autophagy in pancreatic ductal adenocarcinoma[11], retinoblastoma[12], and multiple myeloma[13] to promote tumour progression. However, in this study we found that MALAT1 upregulation in GC could inhibit autophagic flux, which led to Sequestosome1(SQSTM1) protein accumulation and IL6 overexpression. SQSTM1 is a scaffold and stress-inducible protein with multiple domains (such as ZZ, LIR, and PBI), which not only acts as an indicator of autophagy flux but also mediates inflammation response[14]. Hence, SQSTM1 protein accumulation might be responsible for of IL-6 overexpression in GC cells. Majority of studies have revealed the effect of CAFs on GC cell growth or metastasis within the interaction between CAFs and cancer cells. However, the influence on CAFs exerted by GC cells has not been studied in detail. Autophagy inhibition in cancer cells led to expansion and release of cytokines. The dysregulated cytokines could activate the transition from NFs to CAFs via paracrine signalling[15]. Here, we found that impairment of autophagy caused by increased MALAT1 could activate NF to CAF conversion through expansion and secretion of IL-6. These data suggest a critical role for MALAT1 in 
interaction between CAFs and GCs cells. Furthermore, the underlying mechanisms were investigated to identify potential therapeutic strategies targeting GC.

\section{Materials And Methods}

Cell lines. Human GC cell lines MKN-45, MGC-803, and GES-1 were purchased from the Shanghai Institute for Biological Sciences of Chinese Academy of Sciences. GC cells were cultured in RPMI 1640 medium supplemented with $10 \%$ foetal bovine serum (FBS) and penicillin $(100 \mathrm{U} / \mathrm{mL}) /$ streptomycin $(100 \mu \mathrm{g} / \mathrm{mL})$ at $37^{\circ} \mathrm{C}$ in $5 \% \mathrm{CO}_{2}$ in air at saturation humidity.

Isolation and Culture of fibroblasts. CAFs and adjacent NFs were isolated from freshly resected tissues from GC patients at the Department of Surgery, Ruijin hospital affiliated with Shanghai JiaoTong University, School of Medicine. The tissues were well cultured in Dulbecco's modified Eagle's medium (DMEM) with $10 \% \mathrm{FBS}, 100 \mathrm{U} / \mathrm{mL}$ penicillin and $100 \mathrm{ug} / \mathrm{mL}$ streptomycin. A homogeneous group of fibroblasts were developed after two weeks of culture, which were cultured $>10$ times so that the minimum number of clones could be selected. Identification test for CAFs and NFs were performed as described previously (Supplementary Fig.4A囚B).

RNA interference and Plasmids. Small interfering RNAs (siRNAs) that specifically target human MALAT1 and SQSTM1 were purchased from Ribobio Technology (Guangzhou, China) and GenePharma (Shanghai, China), respectively. The siRNAs (100 nM siMALAT1, 100 nM siSQSTM1) were transfected into cells using the RNAi-MAX reagent (Life Technologies, CA, USA) according to the manufacturer's instructions. The pcDNA-MALAT1 plasmid was kindly gifted by Prof. Huating Wang (The Chinese University of Hong Kong, China). Human ELAVL1 expression plasmids were purchased from Sangon Biotech (Shanghai, China). Plasmids $(4 \mathrm{mg} / \mathrm{ml}$ ) were transfected into cells using Lipofectamine 3000 (Life Technologies). Stably transfected cells (MGC-803/MALAT1 $\triangle M G C-803 / N C$ ) were selected by using puromycin ( $1 \mathrm{mg} / \mathrm{ml}$; InvivoGen). The RNA interference sequences are listed in Supplementary Table1.

Quantitative Reverse Transcription PCR (qRT-PCR). Total RNA was extracted with TRIzol $\circledast$ reagent (Invitrogen, Austin, TX, USA), and real-time PCR analysis was conducted according to the manufacturer's instructions (Life Technologies). The mRNA level was measured using the SYBR Green PCR Master Mix (Applied Biosystems, Waltham, MA, USA) and normalized to glyceraldehyde 3-phosphate dehydrogenase (GAPDH) mRNA level. The primer sequences used are listed in Supplementary Table1.

Western blot. Cells were lysed in RIPA buffer containing complete protease and phosphatase inhibitor cocktail (Sigma, USA). The protein concentration of the cell lysates was quantified by a BCA Protein Assay Kit (Pierce, Rockford). The same amount of protein samples was resolved onto $10 \%$ SDS-PAGE and then transferred to PVDF membranes. After blocking with $5 \%$ non-fat milk at $37^{\circ} \mathrm{C}$ for $2 \mathrm{~h}$, the membranes were incubated with the primary antibodies diluted in TBST buffer overnight at $4{ }^{\circ} \mathrm{C}$, followed by incubation with the HRP-conjugated secondary antibody for $2 \mathrm{~h}$ at room temperature. GAPDH antibody was used to verify equal protein loading. The protein band images were captured and analysed 
by a Tanon detection system with ECL reagent (Thermo) and the antigen-antibody reaction was visualized by enhanced chemiluminescence (ECL, Thermo, USA). The antibodies used in this study were obtained from Cell Signaling Technology.

Transfection of mRFP-GFP-LC3 lentivirus vector. The mRFP-GFP-LC3 lentivirus vector was purchased from Genechem (Shanghai, China), which was transfected to GC cells according to the manual. Puromycin $(1 \mu \mathrm{g} / \mathrm{ml})$ was used to select stably expressing mRFP-GFP-LC3 cells. GC cells treated with different plasmids were fixed and analysed using fluorescence microscopy.

Transmission electron microscopy (TEM). GC cells were fixed in $2 \%$ glutaraldehyde containing $0.1 \mathrm{~mol} / \mathrm{I}$ phosphate-buffered saline at $4{ }^{\circ} \mathrm{C}$ for $2 \mathrm{~h}$, incubated in $1 \%$ osmium tetroxide containing $0.1 \mathrm{~mol} / \mathrm{I}$ phosphate-buffered saline for $1.5 \mathrm{~h}$ at $4{ }^{\circ} \mathrm{C}$, dehydrated in graded ethanol, saturated in graded ethanol, embedded, cut into ultrathin sections, stained with lead citrate, and finally viewed using Philip CM-120 TEM (Philips, Netherlands).

RNA stability assay. Transcription inhibitor Actinomycin D (Sigma-Aldrich, USA) was added to culture medium of GC cells transfected with different plasmids for 0, 2, 4, and $6 \mathrm{~h}$. Individual total RNA was harvested for qRT-PCR analysis. The relative mRNA decay rate was measured and fit into an exponential curve.

RNA immunoprecipitation-quantitative PCR (RIP-PCR). RIP assays were performed by using the Magna RIP RNA-Binding Protein Immunoprecipitation Kit (Millipore, USA) according to the manufacturer's instructions. Briefly, cells were lysed in lysis buffer and the cleared lysates were immunoprecipitated with the indicated anti-ELAVL1 and anti-IgG antibodies (Cell Signaling Technology). Immunoprecipitated and input RNA were isolated and reverse transcribed for qRT-PCR amplifications with PTEN 3'-UTR-specific primers. mRNA relative expression level was normalized to input mRNA expression. The primers used for amplification are listed in Supplementary Table1.

Enzyme-linked Immunosorbent Assay (ELISA). The human angiogenesis array (Raybiotech, USA) was used to analyse the soluble mediators according to the manufacturer's protocol. Human IL-6 ELISA kit (Raybiotech) was used to determine the concentration of human IL-6 in medium of different treatment according to the manufacturer's instructions.

Immunofluorescence (IF)/Immunohistochemistry (IHC). For IF assay, GC cells were fixed with $4 \%$ paraformaldehyde for 15 min at room temperature, permeabilized with $0.5 \%$ Triton X-100, and blocked with $5 \%$ BSA for $2 \mathrm{~h}$ before incubation with primary antibodies including anti-ELAV1, anti-DAPI, anti-NFKB, anti-FAP (Cell Signaling Technology), and anti-a-SMA (Abcam, USA) overnight at $4{ }^{\circ} \mathrm{C}$. After incubation with fluorescent secondary antibody for $2 \mathrm{~h}$, images were acquired by fluorescence microscope. Immunohistochemical analysis was conducted as described previously.

Collagen contraction assays. A total of $1 \times 10^{5}$ NFs were suspended in $100 \mu$ DMEM, which was mixed with $100 \mu$ l of collagen mix containing 68.75 $\mu$ DMEM and $31.25 \mu$ Type 1 Rat Tail Collagen (Solarbio, 
China), and added to one well of a $96-$ well plate at $37^{\circ} \mathrm{C}$ for $30 \mathrm{~min}$. After incubation with media derived from different treatments, the gels were photographed and the contractions were evaluated.

Cell-proliferation /EdU assay. Cell viability was measured using Cell Counting Kit-8 (Dojindo, Japan). The cell proliferation and colony-formation assays were performed as described previously. EdU assay was performed using Cell-Light EdU Apollo 567 In Vitro Imaging Kit (Ribobio, Guangzhou, China) according to the manufacturer's instructions.

Xenograft assay. All the experiments were performed in accordance with the official recommendations of the Chinese animal community. Four-week-old male BALB/C nude mice were purchased from the Institute of Zoology, Chinese Academy of Sciences of Shanghai. NFs and MGC-803/MALAT1 or MGC-803/NC cells mixed at the ratio of 1:4 in 100 IL PBS were injected subcutaneously. During the experiment, the tumour volume was measured weekly using the formula $V=\left(\right.$ length $\times$ width $\left.^{2}\right) / 2$.

Statistical methods. Student's t-test or one-way ANOVA were used for statistical analysis when appropriate. All statistical analyses were performed using SPSS 19.0 (SPSS Inc., Chicago, IL, USA). A twotailed value of $\mathrm{P}<0.05$ was considered statistically significant.

\section{Results}

\section{MALAT1 blocks autophagic flux in GC cells}

Anomalous autophagy activity led to variation of inflammation process[16]. The effect of aberrant MALAT1 expression on autophagic flux was investigated. We found that MALAT1 overexpression (Supplementary Fig.1A) could enhance LC3-1 conversion to LC3-II and SQSTM1 protein accumulation in both MKN-45 and MGC-803 cells $\triangle F i g u r e 1 A \otimes P<0.05 \bigotimes$. Contrarily, silencing MALAT1 by transducing siMALAT1 (Supplementary Fig.1B) could inhibit LC3-II and SQSTM1 accumulation (Figure1 A囚P<0.05). Furthermore, MALAT1 had no influence on expression of SQSTM1 mRNA level (Supplementary Fig.1C). LC3-II cloud accumulation results from autophagy activation or reduced turnover from autophagosome to autolysosomes. Moreover, accumulation of SQSTM1 was an indicator of autophagy impairment. Therefore, autophagy inhibitors, 3-methyladenine (3-MA) and bafilomycin A1 (BafA1), were used to treat cells to block autophagy initiation and maturation, respectively. MALAT1 effect on LC3-II and SQSTM1 accumulation was not compromised by 3-MA treatment (Figure 1B, $\mathrm{P}<0.05$ ). In contrast, LC3-II and SQSTM1 accumulation was not affected by BafA1 treatment in MALAT1 overexpression group compared to negative group (Figure $1 C, P<0.05$ ). Subsequently, mRFP-GFP-LC3 lentivirus vector was introduced to determine MALAT1 effect on autophagy flux. When autolysosomes formed, green fluorescence faded, leaving only RFP signal as RFP signal is more stable than green fluorescence in acidic conditions. MALAT1 overexpression in MKN-45 and MGC-803 led to yellow puncta enrichment rather than red ones, indicating autolysosome maturation blockage (Figure 1D, E, $P<0.05$ ). Additionally, we used TEM to evaluate autophagosomes and found out that the number of autophagic vesicles increased in MKN- 
45/MALAT1 and MGC-803/MALAT1 cells compared to that in control cells (Figure 1F, G). Taken together, these results suggested that increased MATLA1 in GC cells could impair autophagy flux.

\section{MALAT1 activates AKT/mTOR pathway to inhibit autophagy in GC cells}

GESA dataset analysis was performed to suggest that autophagy was negatively association with MALAT1 expression (Figure2A, NES=-1.459, FDR q-value=0.06). Activation of mTOR is crucial to inhibit autophagy flux, which led to substantial autophagosome-lysosome fusion inhibition and lysosome dysfunction so that autophagy degradation was impaired[17, 18]. Hence, both phosphorylated-mTOR (pmTOR) and its key substrate, phosphorylated-p70 S6 kinase (p-p70S6K), were detected while assessing MALAT1 effect on mTOR pathway activation. As shown in Figure 3B, a significant p-mTOR and p-p70S6K level increase was observed in MKN-45 and MGC-803 cells transfected with MALAT1 overexpression vectors. Silencing MALAT1 resulted in p-mTOR and p-p70S6K level reduction, which indicated that MALAT1 could activate mTOR pathway (Figure2B, $P<0.05$ ). Autophagy flux impaired by MALAT1 in GC led to SQSTM1 accumulation and rapamycin was used to inhibit mTOR activation to better understand whether MALAT1 impaired autophagy degradation to elevate SQSTM1 accumulation via mTOR pathway. Rapamycin could promote SQSTM1 reduction through silencing and reversing mTOR activation induced by MALAT1 in MKN-45 and MGC-803 cells (Figure $2 \mathrm{C}, \mathrm{P}<0.05$ ). Since canonical PTEN/AKT pathway could regulate mTOR activity, phosphorylated AKT and PTEN expressions were also detected. MALAT1 overexpression could obviously downregulate PTEN protein level and upregulate phosphorylated AKT levels in MKN-45 and MGC-803 cells (Figure 2D, P<0.05). Additionally, we found that MALAT1 not only inhibited PTEN protein level, but also negatively regulated PTEN mRNA expression in MKN-45 and MGC803 cells (Figure $2 E, F, P<0.05$ ). Analysis of GSE dataset (GSE26942) also indicated strong negative correlation between MALAT1 and PTEN mRNA (Figure 2G, P<0.05). Taken together, increased MALAT1 could negatively regulate PTEN expression to activate AKT/mTOR pathway, thus impairing autophagy flux and further elevating SQSTM1 accumulation in GC cells.

\section{MALAT1 inhibits PTEN expression at post-transcriptional level}

Although it has been demonstrated that MALAT1 could inhibit PTEN mRNA expression, the underlying mechanism has not been reported. Several studies have addressed the interaction between MALAT1 and the RNA binding protein (RBP) ELAVL1 to suppress target gene expression via modifying mRNA stability or mRNA initiation[19]. This inspired us to investigate whether MALAT1 regulates PTEN mRNA expression at the post-transcriptional level. The transcription inhibitor actinomycin D (Act D) was added in MKN-45 and MGC-803 cells transfected with or without MALAT1 plasmids for different times ranging from 0 to 6 hours. The levels of remaining mRNAs were determined, and the PTEN mRNA half-lives decreased from $5.62 \pm 0.21$ to $1.54 \pm 0.12 \mathrm{~h}$ and from $5.79 \pm 0.18$ to $2.47 \pm 0.17 \mathrm{~h}(\mathrm{P}<0.01)$ in MKN-45 and MGC-803 cells, respectively, in response to increased MALAT1 (Figure 3A, $\mathrm{P}<0.01$ ). AU-rich elements (AREs) usually exist in the 3'-UTR of mRNA, which could interact with RBPs to modulate mRNA stability. RBPmap database was used to analyse the ARE regions in PTEN 3'-UTR and predict the potential RBPs, which revealed that ARE regions were abundant in PTEN 3'-UTR and most possibly in ARE-ELAVL1 binding regions (Figure 
3B). Meanwhile, significant positive correlation between ELAVL1 and PTEN mRNA expression was observed via analysing GEO datasets (GSE63048) (Figure 3C, $\mathrm{P}<0.001$ ). Moreover, we found that ELAVL1 upregulation could increase PTEN mRNA expression in both MKN-45 and MGC-803 cells (Figure 3D, $P<0.05)$. Similarly, after ELAVL1 overexpression, the PTEN mRNA half-lives increased from $6.16 \pm 0.20$ to $8.13 \pm 0.28 \mathrm{~h}$ and from $5.29 \pm 0.18$ to $8.92 \pm 0.35 \mathrm{~h}$ in MKN-45 and MGC-803 cells, respectively (Figure $3 \mathrm{E}$, $P<0.01)$. These results indicated that ELAVL1 could stabilize the PTEN mRNA. In addition, increased MALAT1 had no influence on ELAVL1 expression in GC cells (Supplementary Fig.2A). Based on the current evidence on the opposite effects of MALAT1 and ELAVL1 on PTEN mRNA expression and the reported correlation between MALAT1 and ELAVL1, we assumed that increased MALAT1 could competitively interact with ELAVL1 to expose PTEN 3'-UTR such that PTEN mRNA destabilization was augmented. To determine the above assumption better, rescue assay was carried out. As shown in Figure 3F, ELAVL1-induced PTEN mRNA levels were significantly abolished by increased MALAT1 in both MKN45 and MGC-803 cells (Figure3F, P<0.05). Subsequently, RIP-PCR assay was performed to determine PTEN 3'-UTR enrichment bound by ELAVL1 with or without MALAT1 transfection in MGC-803 cells (Figure 3G, $H, P<0.05$ ) and MKN-45(Supplementary Fig.2B-D, $P<0.05$ ). These results showed that ELAVL1 could bind more MALAT1 mRNA fractions than PTEN 3'-UTR enrichments under MALAT1 overexpression condition. Additionally, we found that increased MALAT1 led to more ELAVL1 protein being distributed within the nucleus where MALAT1 was located (Figure 3I), indicating that ELAVL1 nucleocytoplasmic shuttling was abrogated and resulted in PTEN mRNA destabilization. The combined data implied that MALAT1 could competitively interact with ELAVL1 to destabilize PTEN mRNA.

\section{Inhibition of autophagy promotes IL-6 secretion via accumulation of SQSTM1}

Increasing evidence showed that autophagy flux inhibition aggravates the inflammatory response[20]. To investigate whether inhibition of autophagic flux inhibited by MALAT1 in GC cells could increase inflammatory cytokine release, human cytokine antibody array was used to compare the conditioned media of MKN-45/MALAT1 and MGC-803/MALAT1 cells and those of MKN-45/NC and MGC-803/NC cells (Supplementary Table1). The significant IL-6 increase was detected in cultured media (CM) of MKN45/MALAT1 and MGC-803/MALAT1 cells (Figure 4A), which was further confirmed by ELISA assay (Figure 4B, P<0.05). Next, we investigated the IL-6 expression in mRNA and protein level within GC cells transfected with MALAT1 plasmids, which demonstrated that increased MALAT1, could promote both IL6 protein and mRNA expressions in MKN-45 and MGC-803 cells (Figure 4C, D, P<0.05). IL-6 expression is regulated by a wide range of transcription factors and NF-KB plays a crucial role. As expected, NF-KB activation and nuclear translocation was observed in MKN-45 and MGC-803 cells transfected with MALAT1 (Figure 4E, F, P<0.05). In addition, increased MALAT1 had no effect on SQSTM1 mRNA expression, which had been shown in first part of results. As mentioned above, increased MALAT1 impaired autophagic flux, resulting in elevated SQSTM1 accumulation within GC cells, with SQSTM1 being involved in both autophagy and inflammation response. Therefore, we assumed that MALAT1 might activate NF-KB pathway to increase IL-6 expression via SQSTM1. SQSTM1 siRNA-treated MKN45/MALAT1 and MGC-803/MALAT1 cells abrogated the enhanced phosphorylated-NF-KB and IL-6 expressions. Similarly, increased SQSTM1 via transfection with SQSTM1 plasmid could reverse NF-KB/IL- 
6 inactivation pathway caused by MALAT1 siRNAs (Figure 4G, $P<0.05$ ). Taken together, increased MALAT1 could elevate SQSTM1 accumulation to activate NF-KB so that IL-6 expression could be increased.

\section{Impairment of autophagy in GC cells facilitates transition from NFs to CAFs}

Existing evidence have revealed that inflammatory cytokines in TME can induce conversion of NFs to CAFs. Thus, autophagic flux impairment-induced inflammatory cytokine effect on NF activation was investigated. We found that the CM collected from MKN-45/MALAT1 and MGC-803/MALAT1 cells markedly induced NFs to acquire myofibroblast phenotype characterized by a-SMA and FAP expression (Figure 5A-C). Furthermore, the fibroblast contraction abilities were markedly enhanced after treatment with CM derived from GC cells with increased MALAT1 (Figure 5D, P<0.05). To determine whether IL-6 was the dominant driver of this effect, NFs were treated with rlL-6, and the results demonstrated a dosedependent FAP and a-SMA expression increase (Figure 5E, $\mathrm{P}<0.05$ ). To better understand the paracrine effect of MKN-45/MALAT1- and MGC-803/MALAT1-secreted IL-6 on fibroblasts, anti-IL-6 neutralizing antibody was used within rescue assay, which could weaken FAP and a-SMA expression in NFs treated with CM from GC cells with increased MALAT1 (Figure 5F, P<0.05). These results demonstrated that autophagy impairment-induced IL-6 from GC cells could activate NF to CAF conversion in a paracrine manner.

To demonstrate the function of activated fibroblast converted from NFs (Activated-NFs), NFs treated with CM derived from GC cells with increased MALAT1 were prepared to perform proliferation assay and EdU dye assay. Then, MKN-45 and MGC-803 cells were incubated by CM collected from fibroblasts pre-cocultured with MKN-45/MALAT1 and MGC-803/MALAT1 cells, respectively. As shown in Figure 6A-C, GC cells exhibited proliferation and colony formation enhancement after treatment (Figure $6 A-C, P<0.05$ ). To further determine whether IL-6 from activated-NFs play crucial role in promoting GC cells proliferation, IL6 blocking antibody was used to treat Activated-NF/MKN-45 and Activated-NF/MGC-803 group. Then we found that impairment of IL-6 with blocking antibody could significantly attenuate GC cells proliferation (Figure 6D, $\mathrm{P}<0.05$ ). Additionally, we further investigated whether activated-NFs could promote tumour growth in vivo. Co-injection of activated-NFs or NFs cells with MGC-803 was performed in nude mice. MGC-803 treated activated-NFs cells generated tumours with larger volume and weight than those generated by MGC-803 treated with NFs (Figure 6E, F, P<0.05). Furthermore, immunohistochemistry staining results showed that the FAP and a-SMA (CAF activation markers), SQSTM1 (autophagy marker) and IL-6 expressions were highly increased in MGC-803/activated-NF group (Figure 6G, $P<0.01$ ), which is consistent with in vitro experiment results.

\section{IL-6 derived from CAFs promote MALAT1 expression in GC cells}

The Cancer Genome Atlas (TCGA) data demonstrated that MALAT1 was highly expressed in GC (Supplementary Fig.3A), and survival curve analysis with GEO dataset showed MALAT1 expression was negatively correlated with post-progression survival time of GC patients (Supplementary Fig.3B). Increasing evidence showed that MALAT1 were aberrantly overexpressed and could act as oncogene in 
GC[21]. The crosstalk between CAFs and GC cells could aggravate dysregulation of gene expression[22, 23]. However囚the mechanism of upregulation of MALAT1 within TME was rarely reported. Therefore, coculture CAFs or NFs with GC cells were performed to determine whether CAFs could upregulate MALAT1 expression in GC cells via paracrine signaling. As shown in figure1A, relative expression of MALAT1 was significantly higher in MKN-45 or MGC-803 co-cultured with CAFs group than that co-cultured with NFs group (Figure7A $\triangle P<0.05$ ), which means cytokine from CAFs might induce MALAT1 expression in GC. GESA dataset analysis was performed to suggest that IL-6/STAT3 pathway signaling was positive association with MALAT1 expression (Figure 7B, NES=1.459, FDR q-value=0.26). Then expression of IL-6 in CAFs, NFs and GC cells were determined by ELISA, which showed that IL- 6 was dominantly overexpressed in CAFs (Figure 7C, $\mathrm{P}<0.01$ ). Furthermore, higher expression of MALTA1 was detected in MKN-45 and MGC-803 cells treated with recombinant IL- 6 protein (rIL-6) than that in MKN-45 and MGC803 cells alone (Figure 7D, P<0.05). Blocking IL- 6 activity with neutralizing IL- 6 antibody of the co-culture system of CAFs and GC cells led to obvious impairment of MALAT1 expression (Figure 7E, P<0.05), indicating IL-6 derived from CAFs could promote MALAT1 expression in GC cells. GEO dataset (GSE60839) analysis showed overexpression of MALAT1 was significantly positive with expression of IL6 and STAT3 in GC samples (Figure 7F, G, P<0.05), suggesting STAT3 might be responsible for highly expression of MALAT1 in GC.

Subsequently, upregulation of STAT3 expression via transfected STAT3 overexpression plasmid led to increasement of MALAT1 in both MKN-45 and MGC-803(Figure 7H, $\mathrm{P}<0.05$ ). Furthermore, WP1066, a selective STAT3 inhibitor (inhibition of efficiency was shown in Supplementary Fig.4C, D), could attenuate MALAT1 expression induced by recombinant IL-6 protein (Figure $7 \mathrm{I}, \mathrm{P}<0.05$ ), which suggested that IL-6 could increase MALAT1 expression via stimulating STAT3 activation. With help of JASPAR database, we found that there are 4 most potential binding sites of STAT3 on MALAT1's promoter accounting for upregulation of MALAT1 in GC (Figure 7J). To better understand whether STAT 3 could interact with MALAT1 promoter, dual luciferase reporter assay was carried out to measure luciferase activity after transfecting of truncations of MALAT1 promoter. The results showed that site\#3(-618bp - 200bp) of MALAT1 promoter contains binding sites which mediated MALAT1 transcription activation induced by STAT3 (Figure 7K, $\mathrm{P}<0.01$ ). Moreover, CHIP assay was performed to show that STAT3 could physically binds to site\#3(-618bp -200bp) of MALAT1 promoter in MKN-45 and MGC-803 cells (Figure 7L, P<0.01). Taken together, aberrant MALAT1 expression was partly attributed to IL-6 derived from CAFs via activation of STAT3 pathway within GC TME. Additionally, we also found that overexpression of IL- 6 was detected in MKN-45 and MGC-803 cells treated with rlL-6 (Supplementary Fig.4E).

\section{Discussion}

In the present study, we showed, for the first time, that increased MALAT1 in GC cells could impair autophagic flux to aggravate IL-6 secretion to activate NF to CAF conversion via paracrine signalling, which resulted in GC cell progression. Increased MALAT1 could destabilize PTEN mRNA to activate AKT/mTOR pathway for blocking autophagic flux, leading to IL- 6 overexpression induced by SQSTM1/NF-KB pathway, and the secreted IL-6 from GC cells stimulate NF to CAF conversion (Figure 8). 
The interaction between GC and stromal cells could cause a positive feedback to foster an inflammatory microenvironment and promote GC progression.

It has been widely reported that increased MALAT1 can promote cancer cell proliferation or migration. Previously $\llbracket$ we had found MALAT1 could function as oncogene to promote proliferation of GC cells[24]. However, MALAT1 effect on the interaction between stromal and cancer cells have been rarely studied. Autophagy is a biological process involved with interaction between different types of cells through production of inflammatory mediators $[6,20]$, which can regulate complex multicellular interactions within TME. Several studies have reported that MALAT1 could promote autophagy in various cancers, including retinoblastoma[13], lung cancer[25], and pancreatic cancer[11]. However, autophagy inhibition caused by increased MALAT1 has been rarely reported and investigated. For autophagy regulation, it is widely accepted that the mammalian target of rapamycin complex 1 (mTORC1) from the autophagy-inhibiting PI3k-Akt pathway[26] and increased MALAT1 could activate PI3K-AKT pathway in numerous cancers including GC[27]. In this study, we demonstrated that increased MALAT1 could inhibit autophagy flux through activating AKT/mTOR pathway. Not only LC3-I to LC3-II protein conversion was increased along with MALAT1 augmentation in GC cells, SQSTM1 protein accumulation was also detected, suggesting LC3 protein conversion resulted from autophagy impairment rather than autophagy induction. Additionally, rescue assays were performed to further confirm that increased MALAT1 could inhibited autophagy flux with 3-MA and BafA1 treatment. Besides AKT/mTOR pathway being activated by increased MALAT1, expression of PTEN, the negative regulator of AKT/mTOR signalling, was also changed. Our study found that increased MALAT1 could destabilize PTEN mRNA to shorten its half-life in GC. AREs were rich in the PTEN 3'-UTR, to which RBP could bind to modulate mRNA stability. ELAVL1 is a ubiquitously expressed RBP that regulates many post-transcriptional steps including mRNA stability and translation. ELAVL1 has been reported to stabilize COX-2, $\beta$-catenin and BECN1 mRNA via binding to target AREs of 3'-UTR[28, 29]. ELAVL1 not only could bind to 3'-UTR, but also interact with IncRNA to form a functional complex. ELAVL1/MALAT1 complex was found to repress CD133 expression and suppress epithelial-mesenchymal transition in breast cancer [19]. However, whether ELAVL1 could bind to PTEN 3'UTR regulating mRNA stability had not been reported, and whether MALAT1 could modulate PTEN mRNA expression via competitive interfering with interaction between ELAVL1 and 3'-UTR was not investigated. In the present study, we found that MALAT1 could interact with ELAVL1 directly and restrain ELAVL1 in the nucleus away from cytoplasm, where it could stabilize PTEN mRNA, as shown by RIP and IF assays. Based on collected evidence, we confirmed that increased MALAT1 could impair autophagy flux in GC via stimulating PTEN/AKT/mTOR signalling pathway. As the consequence of autophagy impairment caused by MALAT1, SQSTM1 accumulation was increased. SQSTM1 has been reported to be a significant activate factor in inflammatory responses[30, 31]through many signalling pathways including stimulating the NF-KB activation[32, 33]. Therefore, we observed whether SQSTM1/NF-KB activation was responsible for IL-6 upregulation induced by increased MALAT1 in GC. From results of rescue assays, we clearly found that SQSTM1 knockdown could reverse NF-KB activation and IL-6 upregulation caused by MALAT1, and restored SQSTM1 could reverse the NF-KB/IL-6 inhibition induced by silencing MALAT1 in GC cells. 
CAFs secret inflammatory mediators to modulate components in TME and changes in TME can also regulate CAF function. We have shown previously that miR-149 can inhibit CAF activation via targeting IL6 expression, which indicated that IL-6 has an important role in CAF activation process[34]. In this study, we found that increased MALAT1 in GC cells results in IL-6 expression and secretion, and IL-6 augmentation activates NF to CAF conversion. The IL- 6 effect on activating NFs was found in GC. IL-6 could also mediate interaction between cancer cells and CAFs not only by supporting tumour cell growth, but also by promoting fibroblast activation in oesophageal cancer[35]. Although IL-6 could stimulate NF to CAF conversion, the underlying molecular mechanisms was rarely known. Most studies attributed that IL-6 mediate microRNA-dependent pathway to CAF activation[36-38], which could not fully describe the underlying mechanisms. The mechanism of cytokines, like IL-6, on stimulating CAF activation should be further investigated. Chronic inflammation leads to NF activation and their conversion into CAFs, producing pro-tumorigenic cytokines, interacting with the cancer cells, and altering their gene expression profile, which result in cancer progression. In this study, activated CAFs induced by IL- 6 could express aSMA, acquire highly contractile phenotype, and functionally, activated CAFs could facilitate GC cell proliferation, which resulted in co-evolution of CAFs with cancer cells. Additionally, MALAT1 has been reported to be aberrantly overexpressed in GC samples; however, the mechanism of upregulation of MALAT1 within TME was rarely reported. The interaction between CAFs and GC cells could aggravate dysregulation of gene expression. We found that CAFs could upregulate MALAT1 expression in GC cells via paracrine signalling. Moreover, IL-6 derived from CAFs might be responsible for high expression of MALAT1 in GC via promoting STAT3 binding to MALAT1 promoter. In this way, the positive feedback loop contributed to a positive feedback to foster an inflammatory microenvironment and promote GC progression.

\section{Conclusion}

Our results indicate that MALAT1 could inhibit autophagic flux and instigate IL- 6 via regulating PTEN/AKT/mTOR and SQSTM1/NF-KB pathways, which convert fibroblasts to CAFs to promote GC progression $\triangle F$ igure8 $\triangle$. However, the mechanism for CAF activation induced by IL- 6 needs to be further investigated. Our study illustrated a new molecular mechanism underlying the interaction between cancer cells and fibroblasts, which may contribute to provide novel prevention and therapeutic strategies for GC.

\section{Abbreviations}

CAF, cancer-associated fibroblasts; NF, normal fibroblasts; TME, tumor microenvironment; RBP, RNA binding protein; GC, gastric cancer; rlL-6, recombinant IL-6 protein; CHIP-PCR, Chromatin immunoprecipitation-quantitative PCR; AREs, AU-rich elements; CM, cultured medium; TEM, Transmission electron microscopy; ELISA, Enzyme-linked Immunosorbent Assay;

\section{Declarations}


Ethical Approval and Consent to participate: All patient samples were obtained with informed consent from Ruijin Hospital, Shanghai Jiao Tong University School of Medicine. All the experiments were performed in accordance with the official recommendations of the Chinese animal community.

Consent for publication $₫$ Not applicable.

Availability of data and materials囚The data sets used and/or analysed during the current study are available from the corresponding author on reasonable request.

Competing interests: No potential conflict of interest was reported by the authors.

Fundings: This work was supported by the National Natural Science Foundation of China Grant No.91529302(BY Liu), NO.81772518, No. 81871904 (ZG Zhu) and No. 81902944(ZQ Wang); and Interdisciplinary Program of Shanghai Jiao Tong University Grant No. YG2017MS58 (C Li), No. ZH2018QNA51(ZQ Wang) and Multicenter Clinical Trial of Shanghai JiaoTong University of medicine NO.DLY201602(ZG Zhu).

Authors' contributions $₫ W Z Q$, WXJ carried out the molecular lab work, participated in data analysis, carried out sequence alignments, participated in the design of the study and drafted the manuscript; ZTQ carried out the statistical analyses; SLP, LBY, ZZG, CL conceived of the study, designed the study, coordinated the study and helped draft the manuscript. All authors read and approved the final manuscript.

Acknowledgements囚Thanks Prof. Hua-ting Wang (The Chinese University of Hong Kong) and Prof. Kannanganattu V. Prasanth (University of Illinois) for providing Human MALAT1 expression vector as a kind gift.

Author details囚Department of Surgery, Shanghai Key Laboratory of Gastric Neoplasms, Shanghai Institute of Digestive Surgery, Ruijin Hospital, Shanghai Jiao Tong University School of Medicine, Shanghai 200025, People's Republic of China.

\section{References}

1. He Q, Fu Y, Tian D, Yan W: The contrasting roles of inflammasomes in cancer.Am J Cancer Res 2018, 8:566-583.

2. LeBleu VS, Kalluri R: A peek into cancer-associated fibroblasts: origins, functions and translational impact.Dis Model Mech 2018, 11.

3. Shiga K, Hara M, Nagasaki T, Sato T, Takahashi H, Takeyama H: Cancer-Associated Fibroblasts: Their Characteristics and Their Roles in Tumor Growth.Cancers (Basel) 2015, 7:2443-2458.

4. Taniguchi K, Karin M: IL-6 and related cytokines as the critical lynchpins between inflammation and cancer.Semin Immunol 2014, 26:54-74.

5. Monkkonen T, Debnath J: Inflammatory signaling cascades and autophagy in cancer.Autophagy 2018, 14:190-198. 
6. Ngabire D, Kim GD: Autophagy and Inflammatory Response in the Tumor Microenvironment.Int J Mol Sci 2017, 18.

7. White E, Karp C, Strohecker AM, Guo Y, Mathew R: Role of autophagy in suppression of inflammation and cancer.Curr Opin Cell Biol 2010, 22:212-217.

8. Qi Y, Zhang M, Li H, Frank JA, Dai L, Liu H, Zhang Z, Wang C, Chen G: Autophagy inhibition by sustained overproduction of IL6 contributes to arsenic carcinogenesis. Cancer Res 2014, 74:37403752.

9. Guo FX, Wu Q, Li P, Zheng L, Ye S, Dai XY, Kang CM, Lu JB, Xu BM, Xu YJ, et al: The role of the LncRNA-FA2H-2-MLKL pathway in atherosclerosis by regulation of autophagy flux and inflammation through mTOR-dependent signaling.Cell Death Differ 2019.

10. Zhu L, Zhu Y, Han S, Chen M, Song P, Dai D, Xu W, Jiang T, Feng L, Shin VY, et al: Impaired autophagic degradation of IncRNA ARHGAP5-AS1 promotes chemoresistance in gastric cancer.Cell Death Dis 2019, 10:383.

11. Li L, Chen H, Gao Y, Wang YW, Zhang GQ, Pan SH, Ji L, Kong R, Wang G, Jia YH, et al: Long Noncoding RNA MALAT1 Promotes Aggressive Pancreatic Cancer Proliferation and Metastasis via the Stimulation of Autophagy.Mol Cancer Ther 2016, 15:2232-2243.

12. Gao D, Lv AE, Li HP, Han DH, Zhang YP: LncRNA MALAT-1 Elevates HMGB1 to Promote Autophagy Resulting in Inhibition of Tumor Cell Apoptosis in Multiple Myeloma.J Cell Biochem 2017, 118:33413348.

13. Huang J, Yang Y, Fang F, Liu K: MALAT1 modulates the autophagy of retinoblastoma cell through miR-124-mediated stx17 regulation.J Cell Biochem 2018, 119:3853-3863.

14. Sanchez-Martin P, Saito T, Komatsu M: p62/SQSTM1: 'Jack of all trades' in health and cancer.FEBS J 2019, 286:8-23.

15. Xu WW, Li B, Guan XY, Chung SK, Wang Y, Yip YL, Law SY, Chan KT, Lee NP, Chan KW, et al: Cancer cell-secreted IGF2 instigates fibroblasts and bone marrow-derived vascular progenitor cells to promote cancer progression.Nat Commun 2017, 8:14399.

16. Matsuzawa-Ishimoto Y, Hwang S, Cadwell K: Autophagy and Inflammation.Annu Rev Immunol 2018, 36:73-101.

17. Senturk M, Lin G, Zuo Z, Mao D, Watson E, Mikos AG, Bellen HJ: Ubiquilins regulate autophagic flux through mTOR signalling and lysosomal acidification.Nat Cell Biol 2019, 21:384-396.

18. Ye B, Wang Q, Hu H, Shen Y, Fan C, Chen P, Ma Y, Wu H, Xiang M: Restoring autophagic flux attenuates cochlear spiral ganglion neuron degeneration by promoting TFEB nuclear translocation via inhibiting MTOR.Autophagy 2019, 15:998-1016.

19. Latorre E, Carelli S, Raimondi I, D'Agostino V, Castiglioni I, Zucal C, Moro G, Luciani A, Ghilardi G, Monti E, et al: The Ribonucleic Complex HuR-MALAT1 Represses CD133 Expression and Suppresses Epithelial-Mesenchymal Transition in Breast Cancer.Cancer Res 2016, 76:2626-2636.

20. Martinez-Outschoorn UE, Whitaker-Menezes D, Lin Z, Flomenberg N, Howell A, Pestell RG, Lisanti MP, Sotgia F: Cytokine production and inflammation drive autophagy in the tumor microenvironment: 
role of stromal caveolin-1 as a key regulator.Cell Cycle 2011, 10:1784-1793.

21. Sun Y, Ma L: New Insights into Long Non-Coding RNA MALAT1 in Cancer and Metastasis.Cancers (Basel) 2019, 11.

22. Ham IH, Lee D, Hur H: Role of Cancer-Associated Fibroblast in Gastric Cancer Progression and Resistance to Treatments.J Oncol 2019, 2019:6270784.

23. Ma Z, Chen M, Yang X, Xu B, Song Z, Zhou B, Yang T: The Role of Cancer-associated Fibroblasts in Tumorigenesis of Gastric Cancer.Curr Pharm Des 2018, 24:3297-3302.

24. Wang J, Su L, Chen X, Li P, Cai Q, Yu B, Liu B, Wu W, Zhu Z: MALAT1 promotes cell proliferation in gastric cancer by recruiting SF2/ASF.Biomed Pharmacother 2014, 68:557-564.

25. Ma J, Wu K, Liu K, Miao R: Effects of MALAT1 on proliferation and apo-ptosis of human non-small cell lung cancer A549 cells in vitro and tumor xenograft growth in vivo by modulating autophagy.Cancer Biomark 2018, 22:63-72.

26. Kim J, Kundu M, Viollet B, Guan KL: AMPK and mTOR regulate autophagy through direct phosphorylation of Ulk1.Nat Cell Biol 2011, 13:132-141.

27. Li J, Cui Z, Li H, Lv X, Gao M, Yang Z, Bi Y, Zhang Z, Wang S, Zhou B, Yin Z: Clinicopathological and prognostic significance of long noncoding RNA MALAT1 in human cancers: a review and metaanalysis.Cancer Cell Int 2018, 18:109.

28. Mrena J, Wiksten JP, Thiel A, Kokkola A, Pohjola L, Lundin J, Nordling S, Ristimaki A, Haglund C: Cyclooxygenase-2 is an independent prognostic factor in gastric cancer and its expression is regulated by the messenger RNA stability factor HuR.Clin Cancer Res 2005, 11:7362-7368.

29. Zhang Z, Yao Z, Wang L, Ding H, Shao J, Chen A, Zhang F, Zheng S: Activation of ferritinophagy is required for the RNA-binding protein ELAVL1/HuR to regulate ferroptosis in hepatic stellate cells.Autophagy 2018, 14:2083-2103.

30. Korhonen E, Piippo N, Hytti M, Hyttinen JMT, Kaarniranta K, Kauppinen A: SQSTM1/p62 regulates the production of IL-8 and MCP-1 in IL-1beta-stimulated human retinal pigment epithelial cells. Cytokine 2019, 116:70-77.

31. Yan XY, Zhang Y, Zhang JJ, Zhang LC, Liu YN, Wu Y, Xue YN, Lu SY, Su J, Sun LK: p62/SQSTM1 as an oncotarget mediates cisplatin resistance through activating RIP1-NF-kappaB pathway in human ovarian cancer cells.Cancer Sci 2017, 108:1405-1413.

32. Moscat J, Diaz-Meco MT, Wooten MW: Signal integration and diversification through the p62 scaffold protein. Trends Biochem Sci 2007, 32:95-100.

33. Janostiak R, Rauniyar N, Lam TT, Ou J, Zhu LJ, Green MR, Wajapeyee N: MELK Promotes Melanoma Growth by Stimulating the NF-kappaB Pathway.Cell Rep 2017, 21:2829-2841.

34. Li P, Shan JX, Chen XH, Zhang D, Su LP, Huang XY, Yu BQ, Zhi QM, Li CL, Wang YQ, et al: Epigenetic silencing of microRNA-149 in cancer-associated fibroblasts mediates prostaglandin E2/interleukin-6 signaling in the tumor microenvironment. Cell Res 2015, 25:588-603. 
35. Karakasheva TA, Lin EW, Tang Q, Qiao E, Waldron TJ, Soni M, Klein-Szanto AJ, Sahu V, Basu D, Ohashi S, et al: IL-6 Mediates Cross-Talk between Tumor Cells and Activated Fibroblasts in the Tumor Microenvironment.Cancer Res 2018, 78:4957-4970.

36. Yao Q, Cao S, Li C, Mengesha A, Kong B, Wei M: Micro-RNA-21 regulates TGF-beta-induced myofibroblast differentiation by targeting PDCD4 in tumor-stroma interaction.Int J Cancer 2011, 128:1783-1792.

37. Erez N, Glanz S, Raz Y, Avivi C, Barshack I: Cancer associated fibroblasts express pro-inflammatory factors in human breast and ovarian tumors.Biochem Biophys Res Commun 2013, 437:397-402.

38. Doldi V, Callari M, Giannoni E, D'Aiuto F, Maffezzini M, Valdagni R, Chiarugi P, Gandellini P, Zaffaroni $\mathrm{N}$ : Integrated gene and miRNA expression analysis of prostate cancer associated fibroblasts supports a prominent role for interleukin-6 in fibroblast activation. Oncotarget 2015, 6:31441-31460.

\section{Figures}


Fig. 1

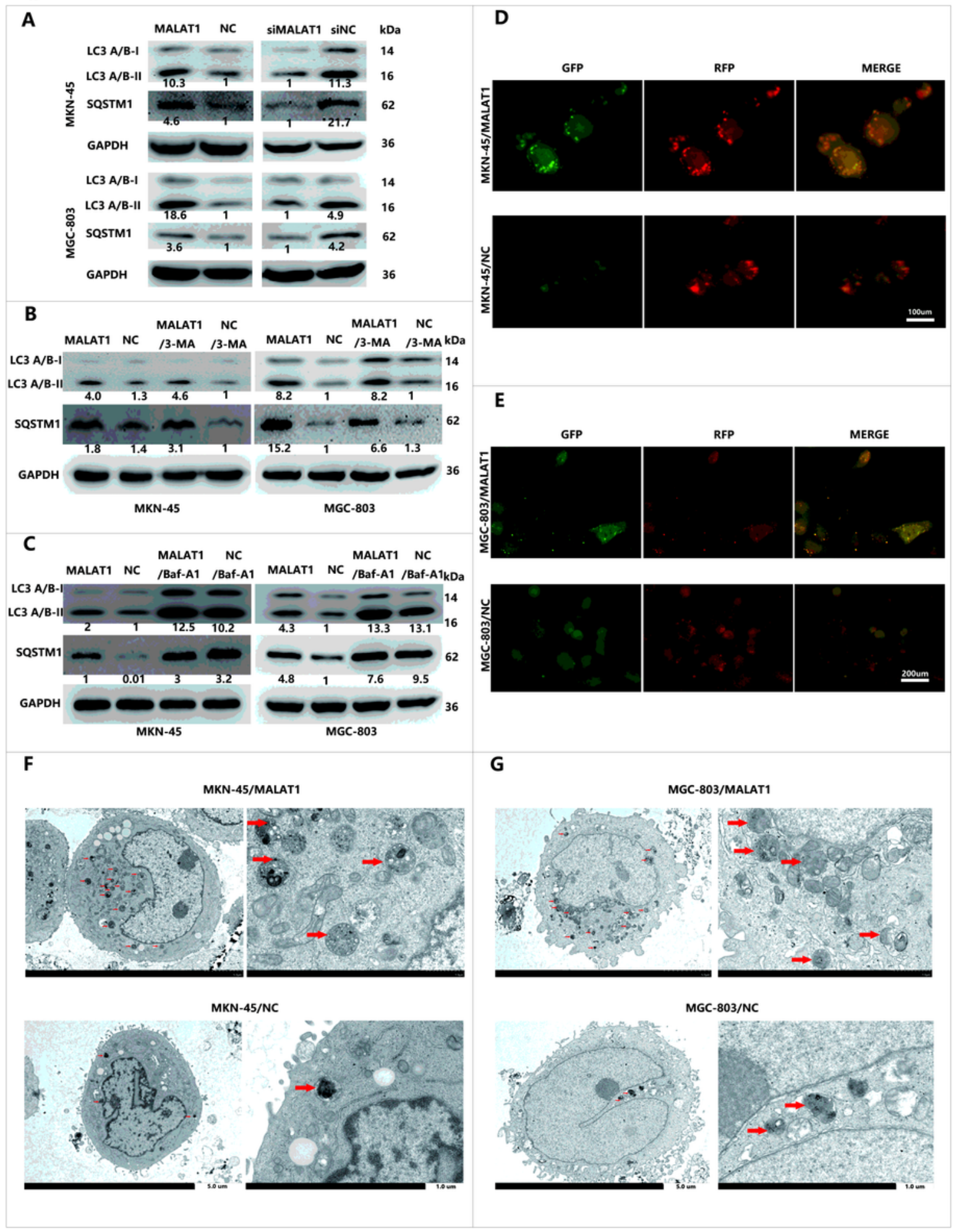

\section{Figure 1}

Increased MALAT1 blocks autophagic flux. A. The LC3 and SQSTM1 protein levels in MKN-45/MALAT1, MGC-803/MALAT1, and their parental cells were determined by western blot assay; B, C. The LC3 and SQSTM1 protein levels in MKN-45/MALAT1 and MGC-803/MALAT1 cells with 3-MA (10 mM) and baf-A1 (10 mM) were determined by western blot assay; D, E. mRFP-GFP-LC3 distribution in MKN-45/MALAT1, MGC-803/MALAT1, and their parental cells were analysed by fluorescence microscopy (MKN-45/MALAT1 


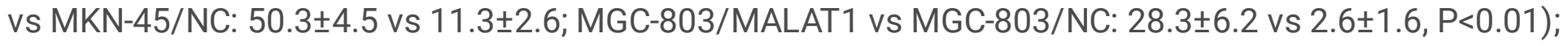
F, G. The number of autophagic vesicles was increased in MKN-45/MALAT1 and MGC-803/MALAT1 group as seen by TEM.

Fig.2

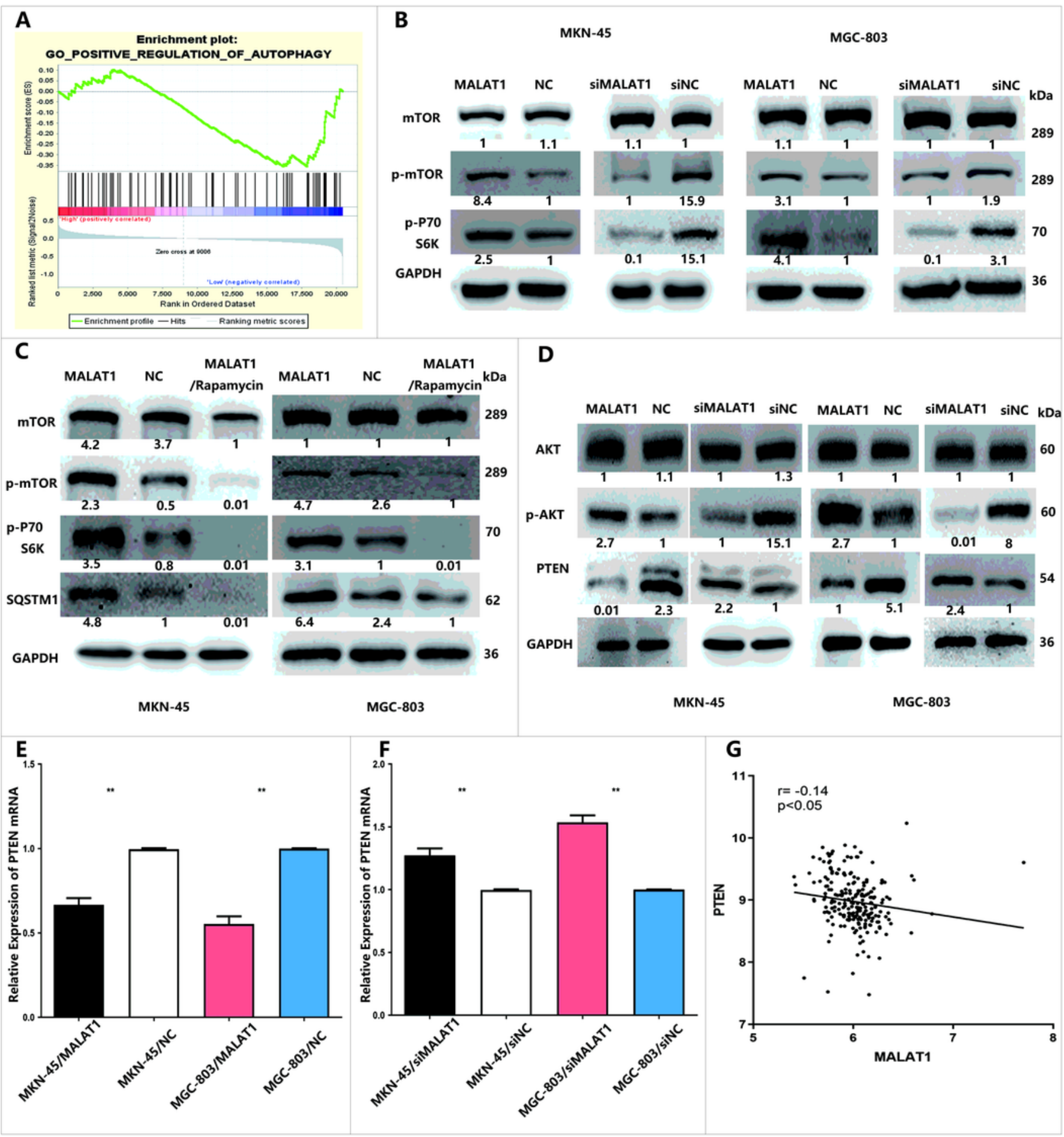

Figure 2

Increased MALAT1 regulated PTEN/AKT/mTOR pathway to inhibit autophagic flux. A. GESA dataset analysis showed negative correlation between MALAT1 and autophagy pathway (NES=-1.459, FDR q- 
value $=0.06) ; B$. The p-mTOR and p-p70S6K protein levels were increased in MKN-45 and MGC-803 cells transfected with MALAT1 overexpression vectors. Silencing MALAT1 resulted in p-mTOR and p-p70S6K level reduction; C. The p-mTOR, p-p70S6K, and SQSTM1 protein levels were detected in MKN-45/MALAT1 and MGC-803/MALAT1 in presence of rapamycin; D. The p-AKT and PTEN protein levels were detected in MKN-45 and MGC-803 cells transfected with MALAT1 overexpression vectors. Silencing MALAT1 resulted in PTEN overexpression and p-AKT downregulation; E, F. The PTEN mRNA levels were detected in MKN45/MALAT1 and MGC-803/MALAT1 cells. Silencing MALAT1 led to PTEN mRNA upregulation (MKN45/MALAT1 vs MKN-45/NC: $0.66 \pm 0.03$ vs 1 , MGC-803/MALAT1 vs MGC-803/NC: $0.53 \pm 0.04$ vs 1, $P<0.01$; MKN-45/siMALAT1 vs MKN-45/siNC: $1.27 \pm 0.04$ vs $1 \pm 0.01$, MGC-803/siMALAT1 vs MGC-803/siNC: $1.53 \pm 0.04$ vs $1 \pm 0.01, P<0.01)$; $G$. GEO dataset analysis showed negative correlation between MALAT1 and PTEN. Bars, S.D.; ${ }^{*} P<0.05 ;{ }^{*} P<0.01$; ${ }^{\star * * P}<0.001$. 
Fig. 3
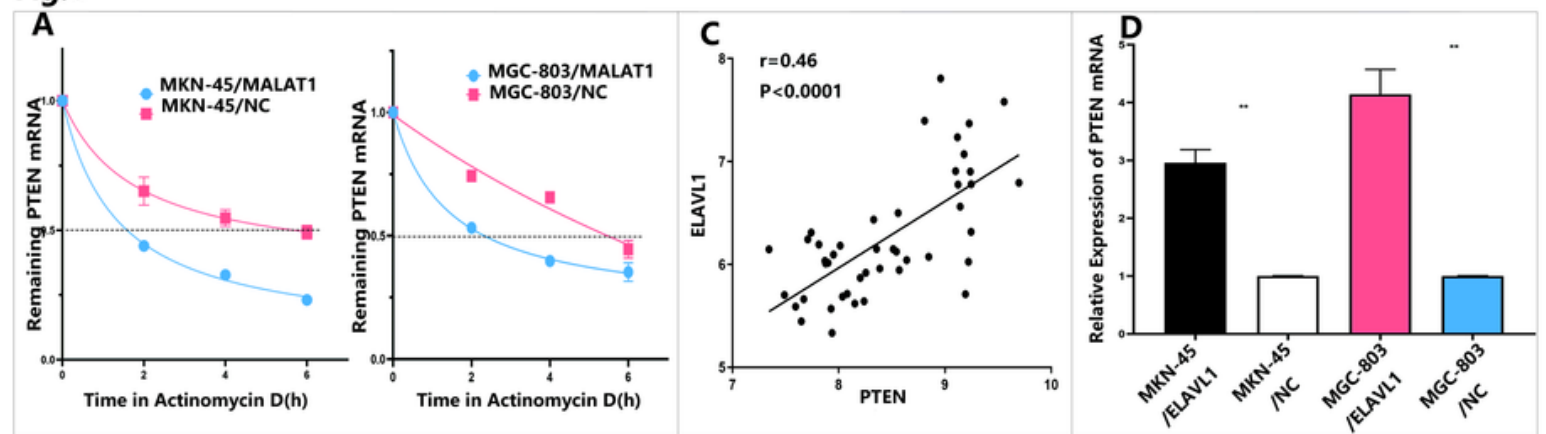

B

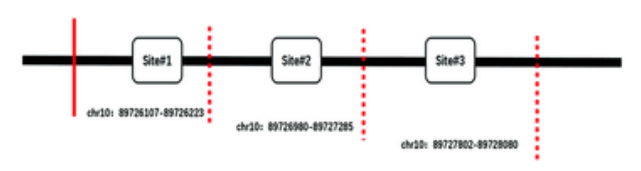

AU-rich elements of PTEN mRNA 3'UTR
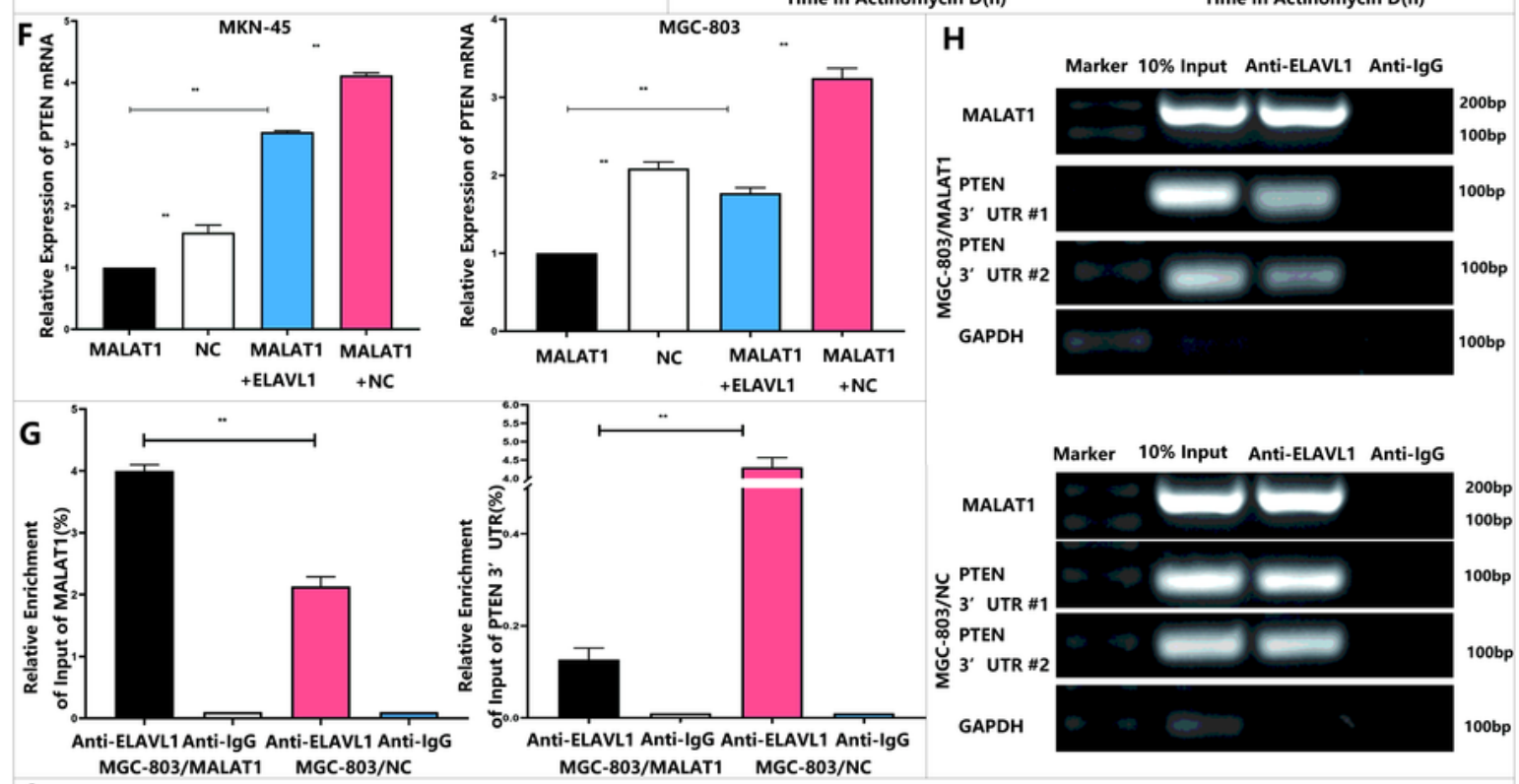
I
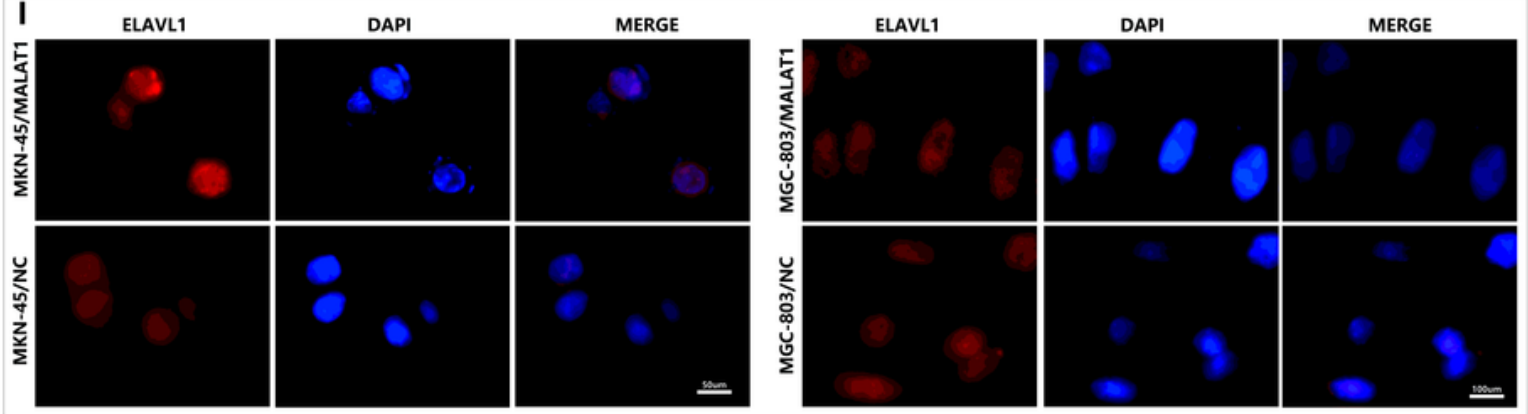

Figure 3

MALAT1 competitively interacted with ELAVL1 to destabilize PTEN mRNA. A. PTEN mRNA expression in MKN-45 and MGC-803 transfected with MALAT1 overexpression vectors and the control after treatment with $5 \mu \mathrm{g} / \mathrm{ml}$ actinomycin D for $0,2,4$, and $6 \mathrm{~h}$. The PTEN transcript half-life was down-regulated by MALAT1; B. Potential bindings sites of AU-rich elements on PTEN 3'-UTR; C. GEO database analysis showed positively correlation between ELAVL1 and PTEN; D. ELAVL1 upregulation increase PTEN mRNA 
in MKN-45 and MGC-803 cells (MKN-45/ELAVL1 vs MKN-45/NC: 2.96 \pm 0.18 vs $1 \pm 0.01$, MGC-803/ELAVL1 vs MGC-803/NC: $4.14 \pm 0.35$ vs $1 \pm 0.01, P<0.01)$; E. PTEN mRNA expression in MKN-45 and MGC-803 transfected with ELAVL1 overexpression vectors and the control after treatment with $5 \mu \mathrm{g} / \mathrm{ml}$ actinomycin D for $0,2,4$, and $6 \mathrm{~h}$. The PTEN transcript half-life was upregulated by ELAVL1 (MKN-45/ELAVL1 vs MKN45/MALAT1+ELAVL1: 1 vs 3.2 \pm 0.02 , MGC-803/ELAVL1 vs MGC-803/MALAT1+ELAVL1: 1 vs $1.80 \pm 0.02, P<0.05) ; F$. The remainder of ELAVL1-induced PTEN mRNA levels were abolished by increased MALAT1 in both MKN-45 and MGC-803 cells; G, H. ELAVL1 captured more MALAT1 mRNA fractions than PTEN 3'-UTR enrichments under MALAT1 overexpression condition through performing RIP-PCR (MALAT1/Anti-ELAVL1 vs NC/Anti-ELAVL1: $4.0 \pm 0.08$ vs 2.13 \pm 0.12 (MALAT1\%), MALAT1/Anti-ELAVL1 vs NC/Anti-ELAVL1: $0.15 \pm 0.04$ vs 4.3 \pm 0.21 (PTEN 3'-UTR\%), P<0.01); I. MKN-45 and MGC-803 cells were transfected with MALAT1 overexpression vectors and the subcellular locations of HuR were determined by immunocytochemistry. Bars, S.D.; ${ }^{*} P<0.05$; ${ }^{*} P<0.01$; ${ }^{* \star * P}<0.001$. 
Fig. 4
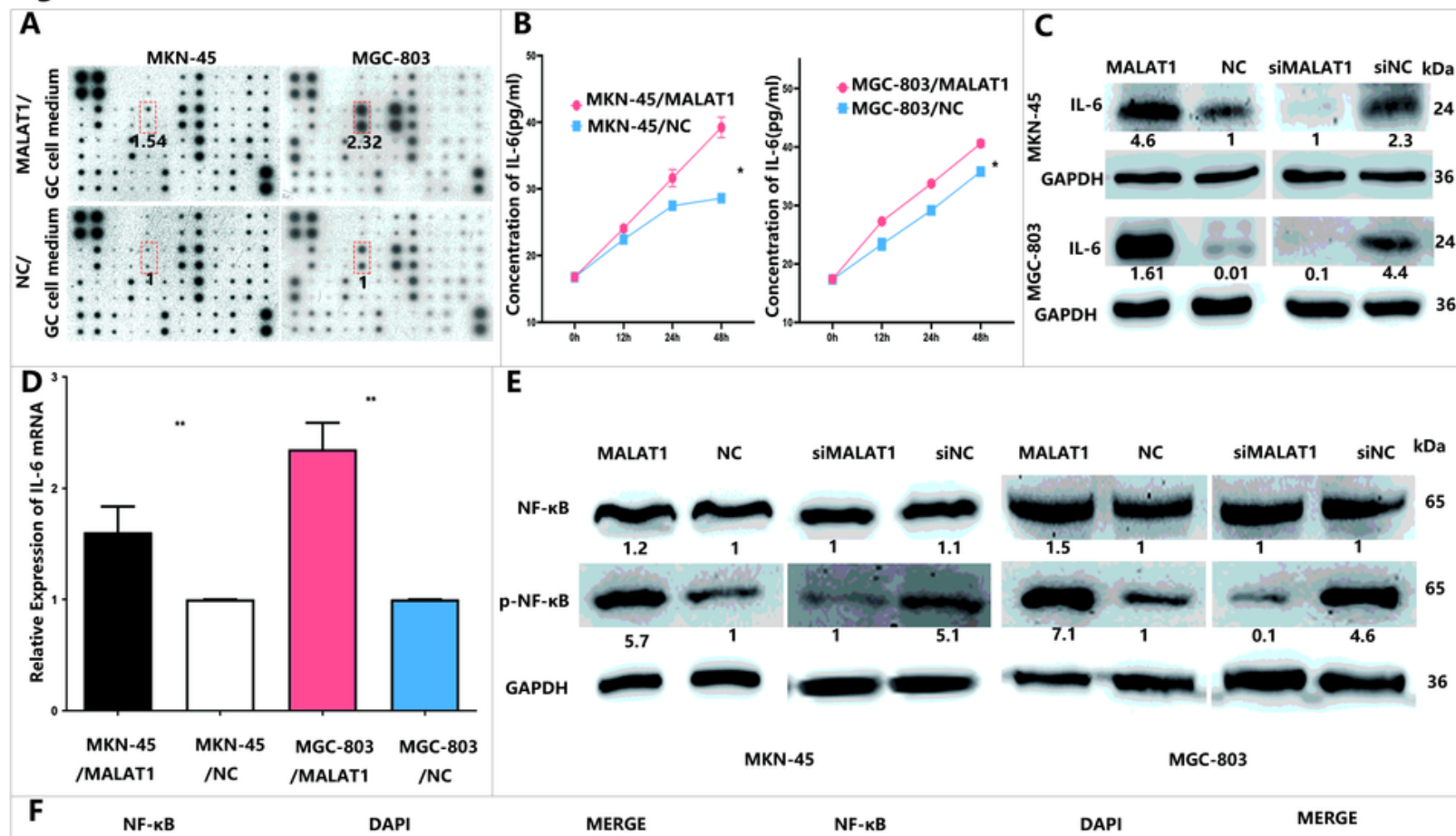

E
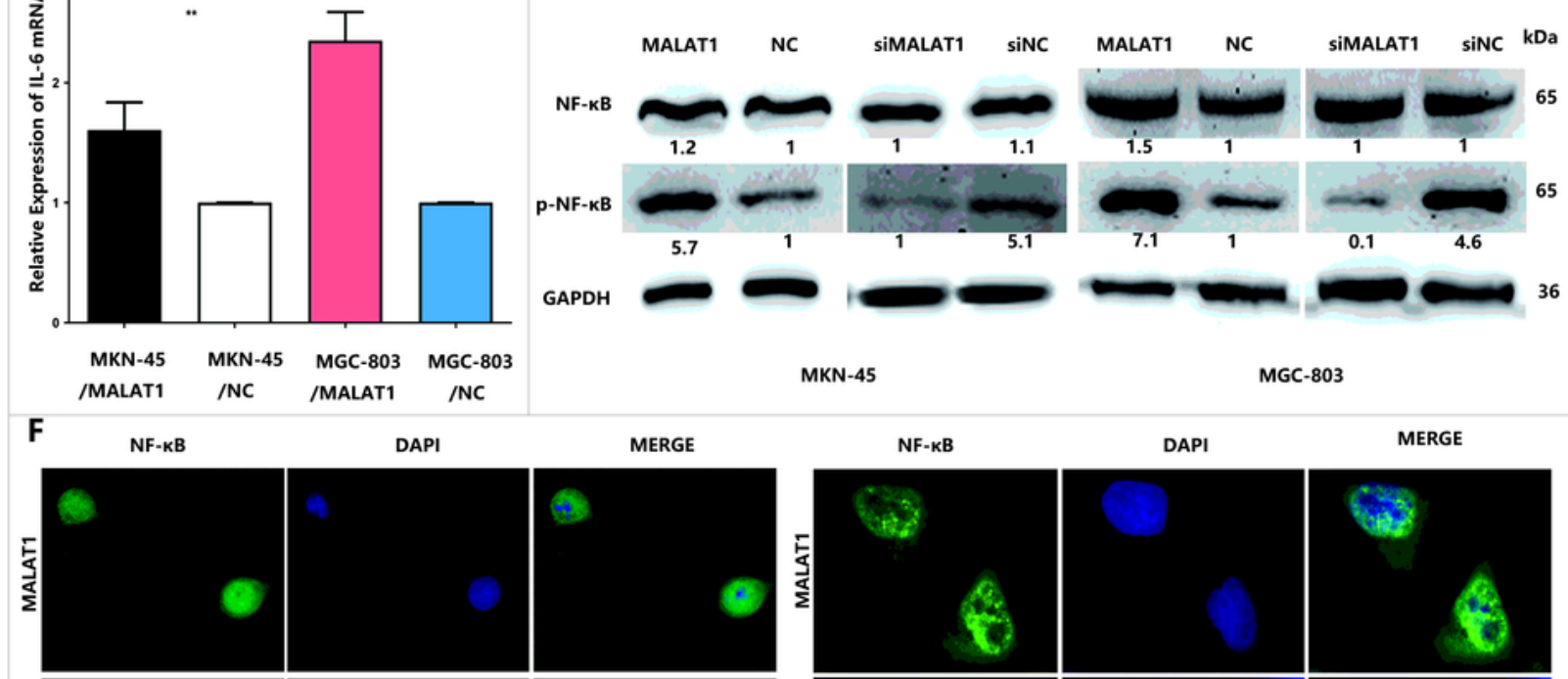

MKN-45

MGC-803
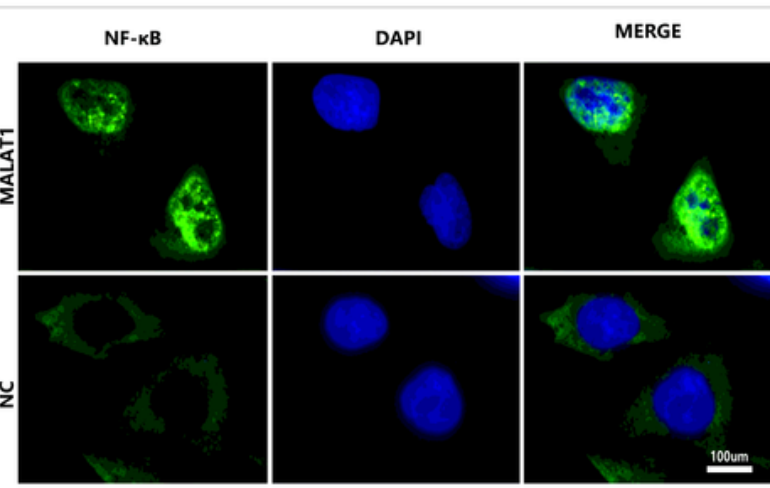

MKN-45

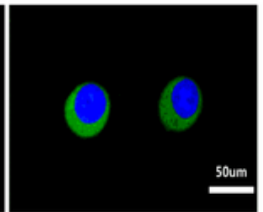

ALAT1

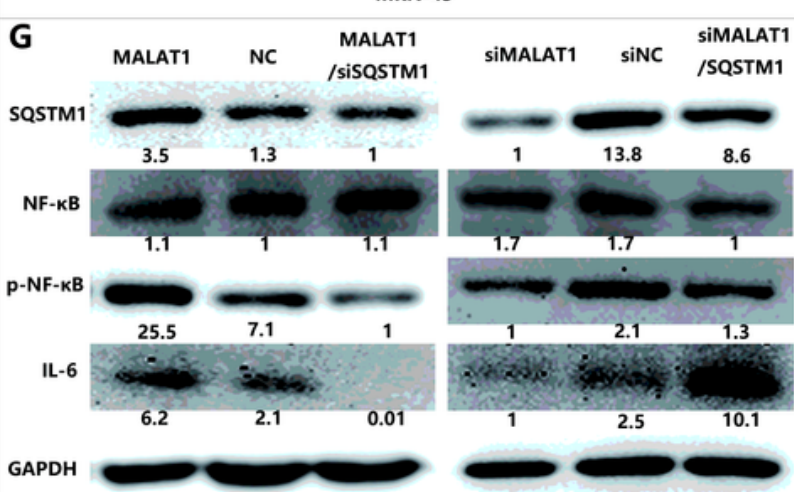

MKN-45

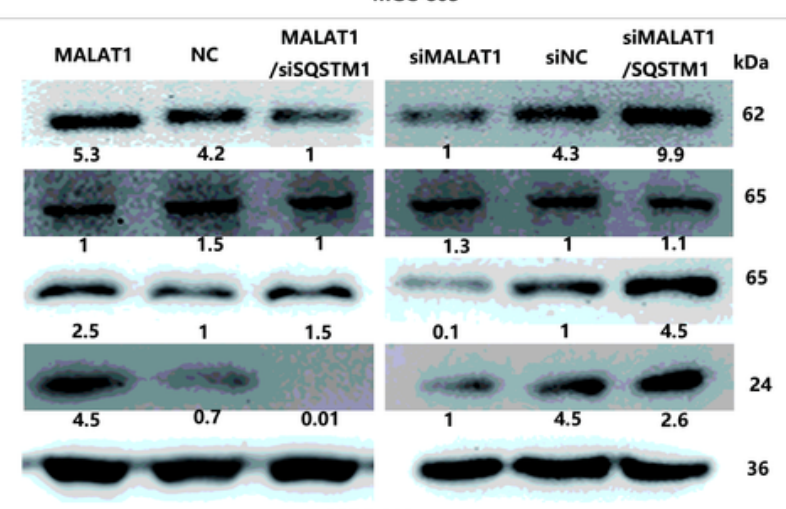

MGC-803

Figure 4

Blockage of autophagy induced IL-6 secretion via SQSTM1/NF-KB pathway activation. A. Human cytokine antibody arrays were used to screen the difference of conditioned medium between GC cells transfected with MALAT1 overexpression vectors and NC vectors; B. IL-6 protein expression level in the MKN-45/MALAT1, MGC-803/MALAT1, and compared groups was quantified $24 \mathrm{~h}$ after changing the culture medium as measured by ELISA (MKN-45/MALAT1 vs MKN-45/NC: 39.24 \pm 1.24 vs 28.62 \pm 0.17 ; 
MGC-803/MALAT1 vs MGC-803/NC: $40.6 \pm 0.47$ vs $35.79 \pm 0.08, P<0.05)$; C, D. The mRNA and protein levels of p-IL-6 were detected in MKN-45 and MGC-803 cells transfected with MALAT1 overexpression vectors. Silencing MALAT1 resulted in IL-6 protein level downregulation (MKN-45/MALAT1 vs MKN45/NC: $1.61 \pm 0.2$ vs $1 \pm 0.07$; MGC-803/MALAT1 vs MGC-803/NC: $2.35 \pm 0.2$ vs $1 \pm 0.07, P<0.01)$; $E$. The NF$\mathrm{KB}$ and $\mathrm{p}-\mathrm{NF}-\mathrm{KB}$ protein levels were increased in MKN-45 and MGC-803 cells transfected with MALAT1 overexpression vectors. Silencing MALAT1 resulted in p-NF-KB level reduction; F. MKN-45 and MGC-803 cells were transfected with MALAT1 overexpression vectors, and the NF-KB subcellular locations were determined by immunofluorescence assay; G. P-NF-KB and IL- 6 expressions were abrogated in MKN45/MALAT1 and MGC-803/MALAT1 cells with SQSTM1 siRNA treatment. Transfected SQSTM1 plasmid into MKN-45 and MGC-803 cells reversed NF-KB/IL-6 pathway inactivation caused by MALAT1 siRNAs. Three biological replicates were performed for in vitro assays. Bars, S.D.; ${ }^{*} P<0.05 ;{ }^{\star \star} P<0.01$; ${ }^{\star \star \star} P<0.001$. 
Fig. 5
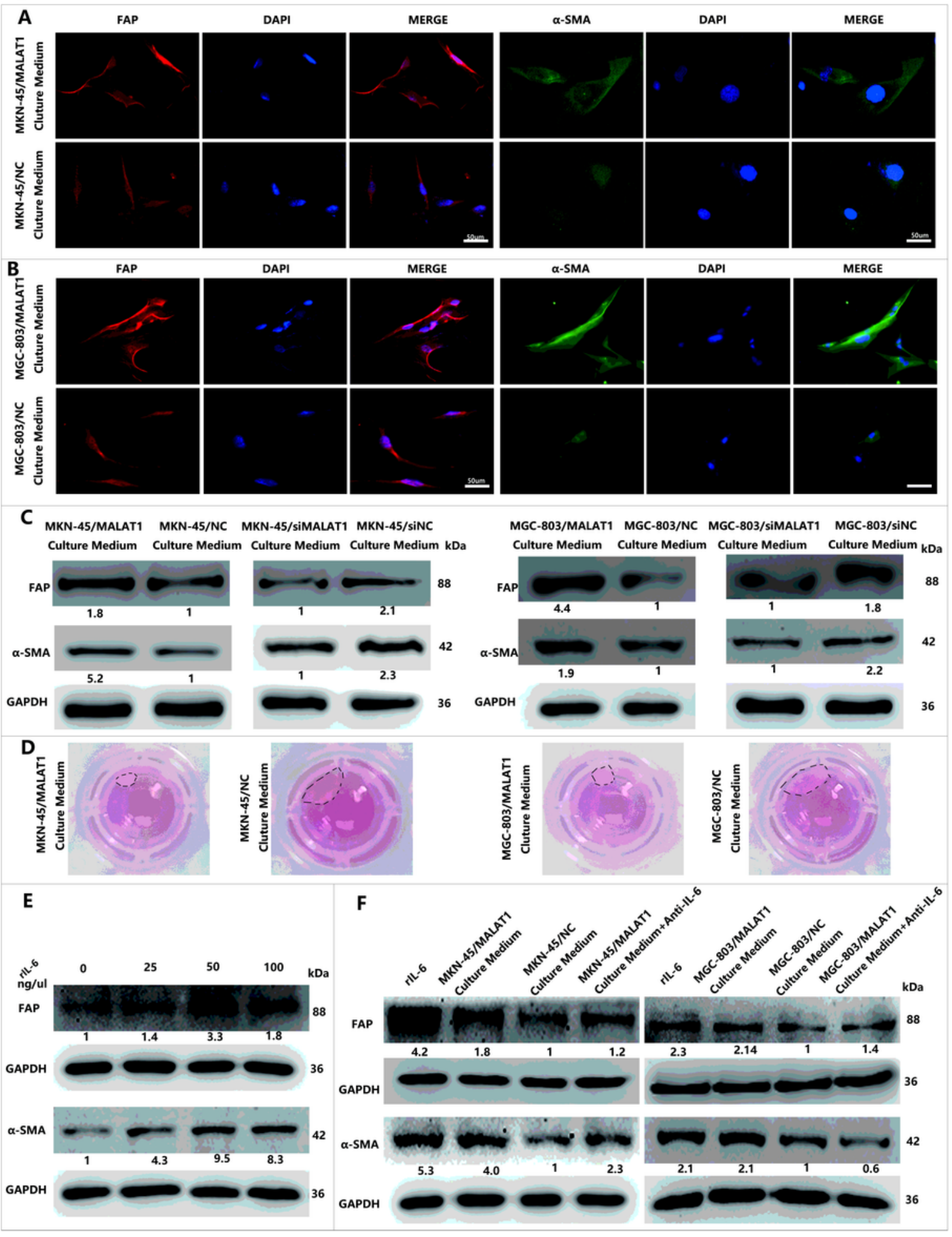

Figure 5

MALAT1-induced IL-6 from GC cells mediates NF-CAF transition. A-C. Cultured medium collected from MKN-45/MALAT1 and MGC-803/MALAT1 cells induced NFs to acquire myofibroblast phenotype characterized by a-SMA and FAP expression as detected by Immunofluorescence and western blot assays; D. NFs treated with cultured medium released by different GC cells or blank control were assessed for their ability to contract collagen; E. The a-SMA and FAP protein levels were detected in NFs 
treated with different concentrations of relL-6; F. Protein levels of a-SMA and FAP in NFs co-cultured with cultured medium collected from MKN-45/MALAT1 and MGC-803/MALAT1 in the presence of IL-6 neutralizing antibody were analysed by western blot. Bars, S.D.; ${ }^{*} P<0.05 ; * \star P<0.01 ; * \star * P<0.001$.

Fig. 6
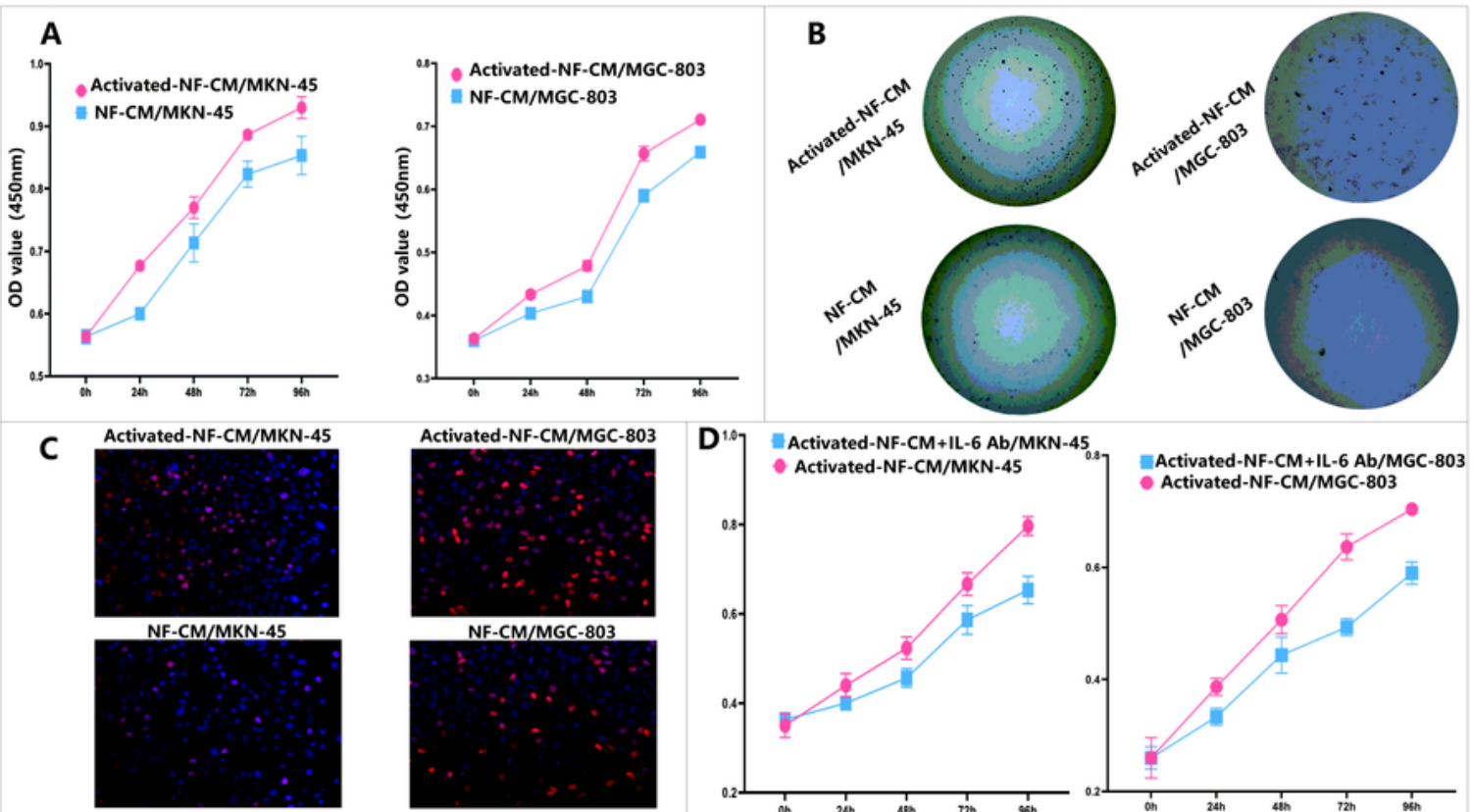

E
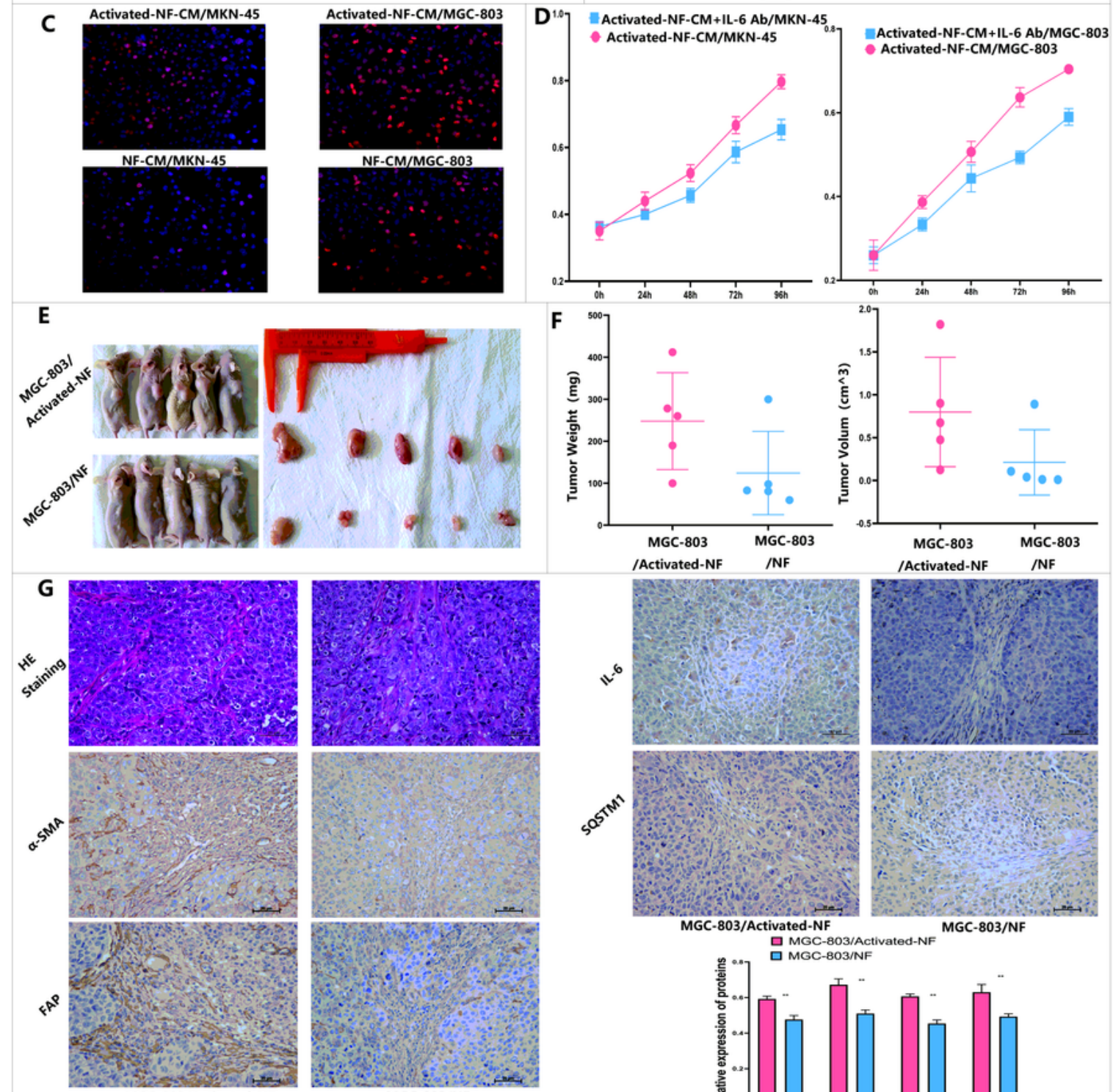

MGC-803/Activated-NF

MGC-803/NF

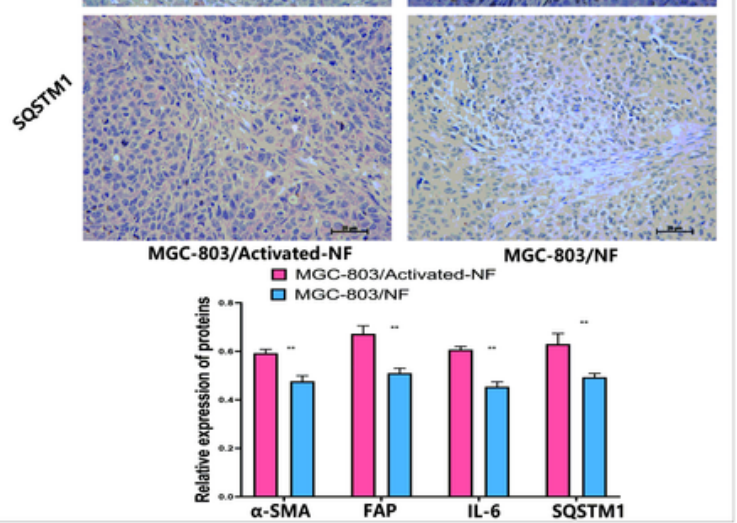

Figure 6

Activated fibroblasts promote GC progression. A-C. Proliferation of MKN-45 and MGC-803 cells treated with activated-NF was determined by CCK8 (Activated-NF-CM/MKN-45 vs NF-CM/MKN-45: 0.93 \pm 0.01 vs 
0.85 \pm 0.02; Activated-NF-CM/MGC-803 vs NF-CM/MGC-803: $0.74 \pm 0.04$ Vs $0.65 \pm 0.01, \mathrm{P}<0.05)$, colonyformation (Activated-NF-CM/MKN-45 Vs NF-CM/MKN-45: 53.5 \pm 11.8 Vs 28 \pm 8.8 ; Activated-NF-CM/MGC803 Vs NF-CM/MGC-803: $37.25 \pm 8.8$ Vs $7.25 \pm 1.9, \mathrm{P}<0.05$ ) and EdU (Activated-NF-CM/MKN-45 vs NFCM/MKN-45: $35.3 \pm 3.8$ vs $12.6 \pm 1.67$; Activated-NF-CM/MGC-803 vs NF-CM/MGC-803: $33.3 \pm 5.4$ vs $18 \pm 2.44, \mathrm{P}<0.05$ ) assays; $\mathrm{D}$. Proliferation of MKN-45 and MGC-803 cells treated with activated-NF-CM and IL- 6 blocking antibody was determined by CCK8 (Activated-NF-CM+IL- 6 antibody/MKN-45 vs ActivatedNF-CM/MKN-45: $0.79 \pm 0.01$ vs $0.65 \pm 0.02$; Activated-NF-CM+ IL-6 antibody /MGC-803 vs NF-CM/MGC-803: $0.703 \pm 0.01$ Vs $0.59 \pm 0.04, P<0.01) \varangle E$. Photographs of tumours in nude mice derived from MGC-803 coinjected with activated-NFs and NFs; F. MGC-803 mixed with activated-NFs generated tumours of larger volume and weight than those generated by MGC-803 mixed with NFs (MGC-803/activated-NFs vs MGC$803 / \mathrm{NFs}: 0.80 \pm 0.5$ vs $0.12 \pm 0.34 \mathrm{~cm}^{\wedge} 3$; MGC-803/activated-NFs vs MGC-803/NFs: $248 \pm 103$ vs $124 \pm 88$ mg, $\mathrm{P}<0.05) ; \mathrm{G}$. FAP, a-SMA, SQSTM1 and IL-6 expressions were examined by IHC in tumours resulting from MGC-803/activated-NFs and MGC-803/NFs group. Bars, S.D.; ${ }^{*} P<0.05 ;{ }^{*} P<0.01 ;{ }^{* \star *} P<0.001$. 
Fig. 7

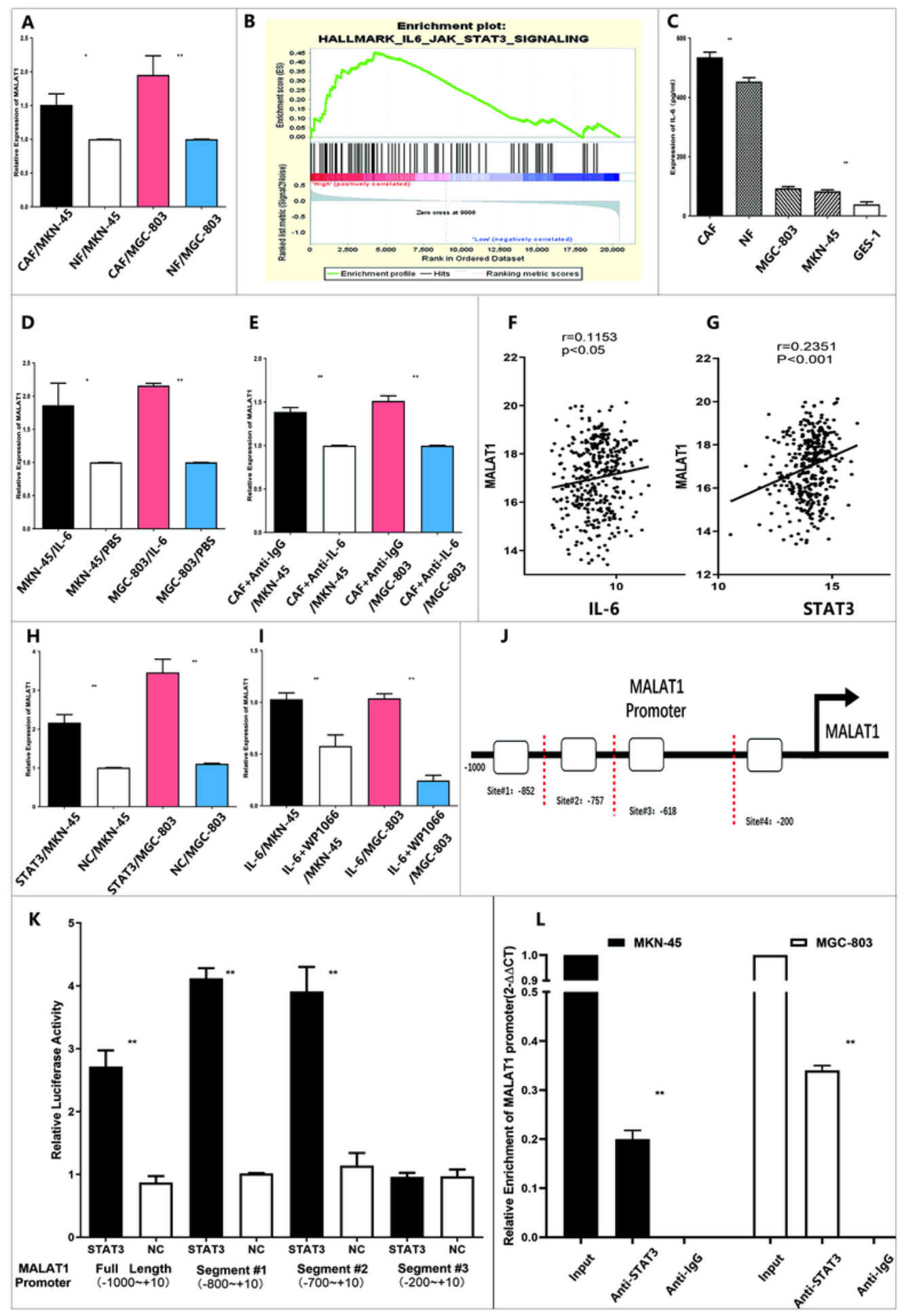

Figure 7

IL-6 derived from CAFs promote MALAT1 expression in GC cells. A. Relative expression of MALAT1 was significantly higher in MKN-45 or MGC-803 co-cultured with CAFs than those co-cultured with NFs (CAF/MKN-45vs NF/MKN-45: $1.51 \pm 0.13$ vs 0.99 \pm 0.01 ; CAF/MGC-803 vs NF/MGC-803: $1.95 \pm 0.23$ vs $\left.0.99 \pm 0.01,{ }^{*} \mathrm{P}<0.05, \star \star \mathrm{P}<0.01\right) ; \mathrm{B}$. GESA dataset analysis showed that IL-6/STAT3 pathway signaling had positive association with MALAT1 expression(NES=1.459, FDR q-value=0.26); C. IL-6 was highly 
expressed in CAFs compared to NFs and GC cells $\left({ }^{*} P<0.05,{ }^{*} \mathrm{P}<0.01\right)$; D. The effect of relL-6 $(100 \mathrm{ng} / \mathrm{mL})$ on MALAT1 expression in MKN-45 and MGC-803 were measured by qRT-PCR (MKN-45/IL-6 vs MKN45/PBS: $1.86 \pm 0.27$ vs $1.03 \pm 0.01$ : MGC-803/IL-6 vs MGC-803/PBS: $2.15 \pm 0.03$ vs $1.03 \pm 0.01, * P<0.05$, $\left.{ }^{*} \mathrm{P}<0.01\right)$; $E$. The effect of CAFs on MALAT1 expression in MKN-45 and MGC-803 cells was determined with the presence of IL- 6 neutralizing antibody or IgG isotype control antibody (CAF+Anti-IgG/MKN-45 vs CAF+Anti-IL-6/MKN-45: $1.38 \pm 0.04$ vs $1.02 \pm 0.03$; CAF+Anti-IgG/MGC-803 vs CAF+Anti-IL-6/MGC-803: $1.51 \pm 0.04$ vs $\left.1.02 \pm 0.03,{ }^{*} \mathrm{P}<0.01\right) ; \mathrm{F}, \mathrm{G}$. GEO dataset analysis suggested MALAT1 expression was positively associated with IL-6 and STAT3 expressions, respectively; H. STAT3 upregulation promoted MALAT1 expression in MKN-45 and MGC-803 cells (STAT3/MKN-45 vs NC/MKN-45: 2.16 \pm 0.16 vs 1: STAT3/MGC-803 vs NC/MGC-803: $3.46 \pm 0.27$ vs $1.1 \pm 0.01$, P<0.01); I. WP1066, a selective STAT3 inhibitor, could attenuate MALAT1 expression induced by recombinant IL- 6 protein, which was measured by qRT-

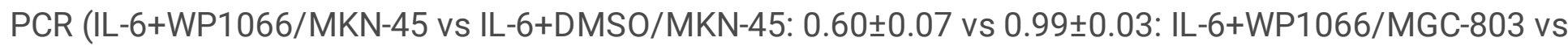
IL-6+DMSO/MGC-803: $0.24 \pm 0.01$ vs $0.99 \pm 0.03, P<0.01$ ); J. Potential STAT3 bindings sites on MALAT1 promoter; K. Luciferase activity was measured after transfecting with MALAT1 promoter truncations, indicating that MALAT1 promoter site\#3 contains binding sites (STAT3 vs NC: $3.91 \pm 0.33$ Vs $1.13 \pm 0.17$, $P<0.01)$; L. Chip assay was performed to show that STAT3 could physically bind to MALAT1 promoter site\#3 in MKN-45 and MGC-803 cells. Bars, S.D.; ${ }^{*}<0.05 ;{ }^{* \star P}<0.01 ; * \star * P<0.001$. 
Fig.8

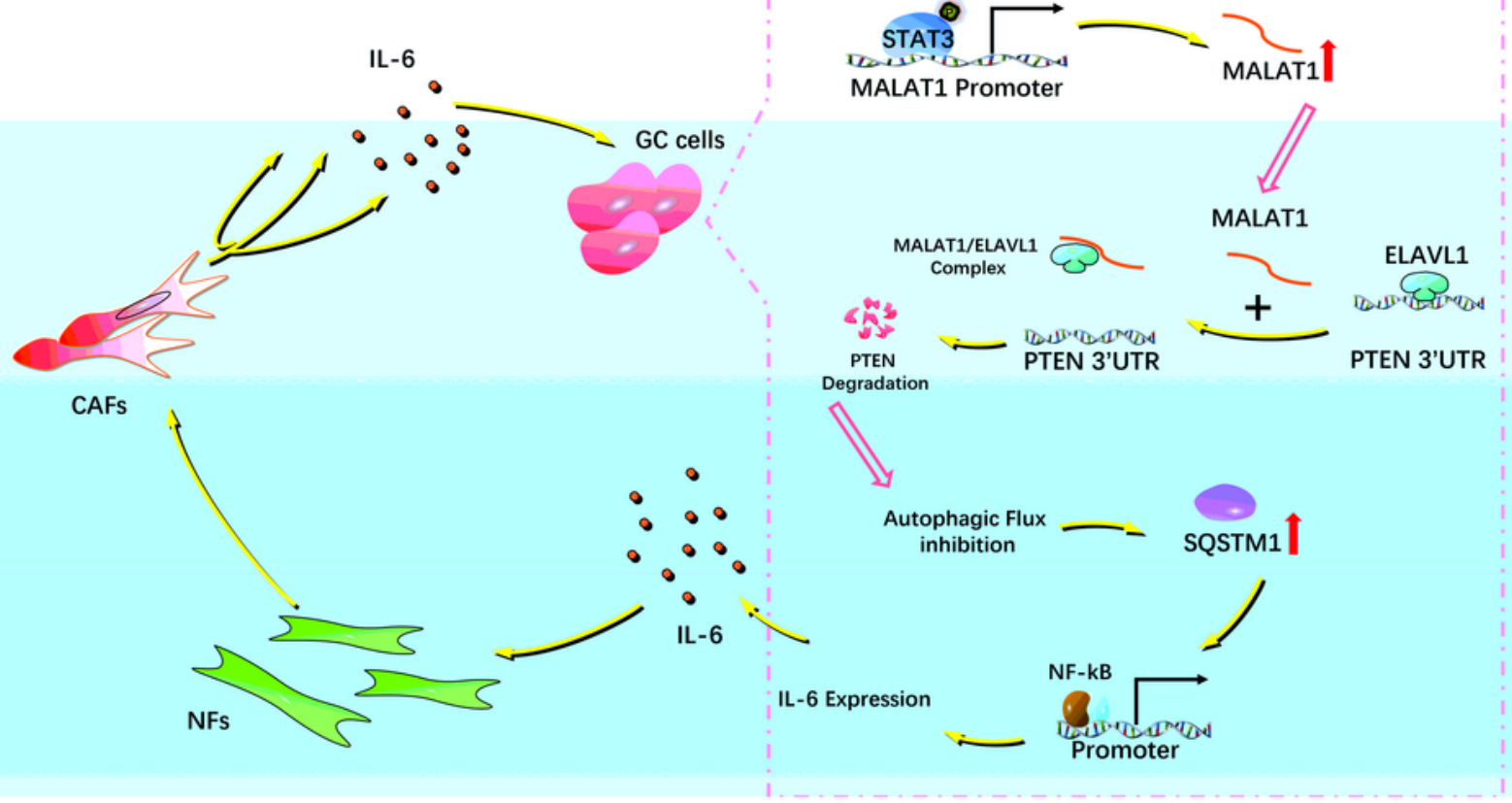

\section{Figure 8}

Schematic diagram summarizing the regulatory role of the MALAT1 in interaction between GC cells and stromal cells. IL-6 secreted from CAFs activated STAT3 binding to MALAT1 promoter to increase MALAT1 expression in GC cells within TME. Increased MALAT1 in GC cells could impair autophagic flux to aggravate IL- 6 secretion to activate converts NFs to CAFs via paracrine signalling, which resulted in GC cell progression. Increased MALAT1 could destabilize PTEN mRNA stability to activate AKT/mTOR 
pathway, which blocked autophagic flux leading to IL-6 overexpression induced by SQSTM1/NF-KB pathway. In addition, IL-6 secretion from GC cells stimulates NF conversion to CAFs.

\section{Supplementary Files}

This is a list of supplementary files associated with this preprint. Click to download.

- SupplementaryTable1.pdf

- SupplementaryFig.4.jpg

- SupplementaryFig.3.jpg

- SupplementaryFig.2.jpg

- SupplementaryFig.1.jpg 